J. DIFFERENTIAL GEOMETRY

55 (2000) 43-82

\title{
DISTRIBUTION OF RESONANCES FOR ASYMPTOTICALLY EUCLIDEAN MANIFOLDS
}

\author{
JARED WUNSCH \& MACIEJ ZWORSKI
}

\begin{abstract}
In this paper we discuss meromorphic continuation of the resolvent and bounds on the number of resonances for scattering manifolds, a class of manifolds generalizing Euclidian $n$-space. Subject to the basic assumption of analyticity near infinity, we show that resolvent of the Laplacian has a meromorphic continuation to a conic neighborhood of the continuous spectrum. This involves a geometric interpretation of the complex scaling method in terms of deformations in the Grauert tube of the manifold. We then show that the number of resonances (poles of the meromorphic continuation of the resolvent) in a conic neighborhood of $\mathbb{R}_{+}$of absolute value less than $r^{2}$ is $\mathcal{O}\left(r^{n}\right)$. Under the stronger assumption of global analyticity and hyperbolicity of the geodesic flow, we prove a finer, Weyl-type upper bound for the counting function for resonances in small neighborhoods of the real axis. This estimate has an exponent which involves the dimension of the trapped set of the geodesic flow.
\end{abstract}

\section{Introduction and statement of results}

Resonances replace discrete spectral data for operators on non-compact domains and are defined as poles of the meromorphic continuation of the resolvent. They are related to the long time behaviour of the wave equation and, in physics, to the existence of states with rest energies and rates of decay given by the real and imaginary parts of resonances — see [33] for an elementary survey and references.

In this paper we consider a class of manifolds generalizing Euclidean $n$-space and we prove meromorphic continuation of the resolvent of the Laplacian to conic neighbourhoods of the continuous spectrum. This is done under the basic assumption of analyticity near infinity. We give an upper bound $\mathcal{O}\left(r^{n}\right)$ on the number of resonances, that is, poles of

Received May 5, 1999. 
the meromorphically continued resolvent, with absolute value less than $r^{2}$.

Under a stronger assumption of global analyticity and hyperbolicity of the geodesic flow, finer estimates are possible. Proceeding in the spirit of [23] and [32] we prove that the counting function for resonances in small neighbourhoods of the real axis satisfies a Weyl type estimate with the exponent related to the dimension of the trapped set of the geodesic flow, $K \subset T^{*} X$, and the size of the neighbourhood. In particular, when we count in a fixed strip of width $r$, the bound is essentially given by $C r^{m / 2}, m=\operatorname{dim} K$, which (very weakly) generalizes the standard Weyl estimate on the number of eigenvalues for a compact manifold, $X$, where $K=T^{*} X$.

The assumptions referred to in the statements of the following theorems will be discussed in detail in Section 2. Manifolds with boundary endowed with scattering metrics [14],[15], generalize asymptotically Euclidean cones. The boundary of such a manifold should be thought of as the boundary of the compactified infinite conic end.

Theorem 1. Let $X$ be a manifold with boundary which is real analytic near $\partial X$, with the scattering metric holomorphic near $\partial X$ in a conic neighbourhood of $X$ in its Grauert tube with base at the Grauert tube of $\partial X$. Then for $\theta_{0}>0$ sufficiently small, the resolvent, $R(z)=$ $(\Delta-z)^{-1}$, continues meromorphically from $\{z: \operatorname{Im} z<0\}$ to $\{z:$ $\left.\arg z<2 \theta_{0}\right\}$ as an operator

$$
R(z): \mathcal{C}_{c}^{\infty}\left(X^{\circ}\right) \longrightarrow \mathcal{C}^{\infty}\left(X^{\circ}\right) .
$$

The poles are of finite rank and their multiplicities are given by

$$
m_{X}(z)=\operatorname{rank} \int_{\gamma} R(\widetilde{z}) d \widetilde{z}, \quad[0,2 \pi) \ni t \mapsto \gamma(t)=z+\epsilon e^{i t}, \quad 0<\epsilon \ll 1 .
$$

Resonances are defined as poles of the meromorphic continuation. The following theorem establishes the natural bound on their number:

Theorem 2. Let $X$ be a scattering manifold satisfying the assumptions of Theorem 1 . For $\theta_{0}>0$ sufficiently small, the counting function of the poles of $R(z)$ satisfies

$$
\sum_{\substack{1<|z|<r^{2} \\ \arg z<\theta_{0}}} m_{X}(z) \leq C r^{n} .
$$


This generalizes to this setting the Euclidean estimates of Melrose, Sjöstrand, Vodev and the second author (see [25] and [33] for references). When a neighbourhood of infinity is isometric to $\mathbb{R}^{n} / \Gamma, \Gamma \subset G L(n)$, finite, then global upper bounds were obtained by Edward [4].

Theorem 2 is optimal, as can already be seen from many Euclidean examples. We can, however, state a result which holds in great generality. The methods presented in Section 3 below allow a generalization of the results of Sjöstrand [25] needed in the argument of Tang and the second author [30] on the existence of resonances (inspired by earlier results of Stefanov and Vodev). Using a refinement of the counting argument of that paper given by Stefanov [29], and the KAM-based quasimode theory of Lazutkin and Popov (see [18] and references given there) one can prove

Theorem 3. Let $X$ be a scattering manifold satisfying the assumptions of Theorem 1 . If there exists an elliptic non-degenerate closed orbit of the geodesic flow on $X$ then for any $N>0$

$$
\sum_{\substack{1<|z|<r^{2} \\ \operatorname{Im} z<|z|^{-N}}} m_{X}(z) \geq r^{n} / C_{N} .
$$

For the proof we refer to [25],[30], and [29] with the needed modifications provided in Section 3 below.

When the flow is hyperbolic we obtain finer upper bounds but no corresponding lower bounds. In the Euclidean case the following theorem is a special case of semiclassical results of Sjöstrand [23] and for $X=\Gamma \backslash \mathbb{H}^{2}$, non-compact with no cusps, it was proved by the second author [32]. A very weak example showing its optimality is given in Section 2. Obtaining lower bounds and generalizations to higher dimensional hyperbolic-like spaces present outstanding problems.

Theorem 4. Let $X$ be a real analytic scattering manifold satisfying the assumptions of Theorem 1. Let $K \subset T^{*} X$ be the trapped set of the geodesic flow on $T^{*} X$ and let us assume that the geodesic flow is hyperbolic on a neighbourhood of $K$. Then for $\alpha>0, a, b>0$, and any $\epsilon>0$,

$$
\begin{gathered}
\sum_{\substack{1<|z|<r^{2} \\
\operatorname{Im} z<a|z|^{\alpha}+b}} m_{X}(z) \leq C_{\epsilon} r^{(1-\alpha) \frac{m}{2}+\alpha n+\epsilon}, \\
\text { where } m=\text { Minkowski dimension of } K .
\end{gathered}
$$


When $K$ is of pure dimension then $\epsilon$ can be taken to be 0 .

The assumption about the flow can be fulfilled when all sectional curvatures are negative on a compact neighborhood of $\pi(K)$.

The Minkowski dimension is defined as follows: for a bounded set $L \subset \mathbb{R}^{m}$ we set

$$
\operatorname{dim} L=m-\sup \left\{d: \varlimsup_{\epsilon \rightarrow 0} \epsilon^{-d}\left(\operatorname{vol}\left\{x \in \mathbb{R}^{m}: d(x, L)<\epsilon\right\}\right)<\infty\right\} .
$$

The set $L$ is said to be of pure dimension if the supremum in (1.1) is achieved. Since $K$ is homogeneous under the natural $\mathbb{R}_{+}$-action on $T^{*} X$, we can apply this definition to the intersection of $K$ with the cosphere bundle.

The starting point of the paper is the proof of Theorem 1. We observe that the complex scaling method of mathematical physics (see Section 3 and references given there) generalizes to the case of asymptotically Euclidean manifolds which are analytic near infinity. The complex dilatation is replaced by a deformation of the manifold inside its Grauert tube: roughly speaking the boundary at infinity is kept fixed and we move the manifold into its complex neighbourhood near that boundary (see Figure 1). The gain of ellipticity thus achieved is particularly clear in the scattering calculus (which we discuss in an appendix in both its classical and semiclassical guises).

The adaptation of methods of Helffer and Sjöstrand [8], [23] to manifolds was carried out in [24] and [32] and we can use those techniques to prove Theorems 2 and 4 . The situation is much clearer than in [32], however, as there is no need for phase space scaling near infinity, which is the main difficulty in the hyperbolic case. Roughly speaking, complex scaling provides the needed gain in ellipticity near infinity so that the microlocal scaling based on the FBI transformation is used a neighbourhood of the trapped set only. That allows us to avoid the development of global FBI transformations generalizing the work of Helffer-Sjöstrand from $\mathbb{R}^{n}$ to scattering manifold: near infinity we proceed more in the spirit of [27] than [8]. The general philosophy is described in Section 2 of $[32]$.

Finally, we remark that in the semi-classical setting of [23], a lower bound of the same form as the upper bound in Theorem 4 has been recently verified numerically [13]. Hence we should expect the analogue of Theorem 3 to hold as well. 
Acknowledgments. We would like to thanks Mike Christ for helpful discussions and in particular for explaining the existence of an analytic defining function of the boundary. Thanks go also to Rafe Mazzeo for suggesting [12] as a source of examples of manifolds satisfying assumptions of Theorem 4, and to Edith Mooers for helpful comments on the manuscript. The first author gratefully acknowledges the support of an NSF Postdoctoral Fellowship; the second author thanks the National Science and Engineering Research Council of Canada, and the National Science Foundation of the U.S. for partial support.

\section{Geometric assumptions}

We assume that $X$ is a compact real analytic $n$-manifold with with a real analytic boundary $\partial X$. Let $X^{\sharp} \supset X$ be an open analytic manifold of the same dimension as $X$, without boundary. By taking the Grauert tube of $X^{\sharp}$ (see [6]) we obtain an open complex manifold $\widetilde{X}$, with $\operatorname{dim}_{\mathbb{C}} \widetilde{X}=n$, such that $X \subset \widetilde{X}$ is a totally real submanifold. That $X$ is totally real means that for $m \in X^{\circ}$ we have

$$
T_{m} X \cap \mathcal{J} T_{m} X=\{0\}
$$

where $T_{m} X \subset T_{m} \widetilde{X}$, and $\mathcal{J}: T_{m} \widetilde{X} \rightarrow T_{m} \widetilde{X}$ gives the complex structure. By abuse of notation we let $\widetilde{X}$ equal $X$ outside of a neighbourhood of $\partial X$ in the case the analyticity assumption is local near $\partial X$.

Using the results of [6] (see Sections 5.2 and 5.3 of [11]), we can find a holomorphic embedding of any open precompact subset of $\widetilde{X}$ into $\mathbb{C}^{N}$ for some $N$. Then, by using the usual metric on $\mathbb{R}^{2 N}$ we can obtain a real analytic Riemannian metric on a neighbourhood of $X$ in $X^{\sharp}$. We can then use it to obtain global (near $\partial X$ ) real analytic normal geodesic coordinates. In particular, we obtain a defining function $x$ of $\partial X$ :

$x \uparrow_{\partial X}=0, \quad d x \uparrow_{\partial X} \neq 0, \quad x: X \longrightarrow[0, \infty)$ is analytic near $\partial X$.

For the meromorphic continuation of the resolvent and for the bounds on the number of resonances in small conic neighbourhoods, we need only make an analyticity assumption near $\partial X$. In this case we think of $\widetilde{X}$ as a complex neighbourhood of $X$, containing a neighbourhood of $\partial X$ in $X$ as a totally real submanifold of maximal dimension.

Thus, we can write

$$
\begin{gathered}
X=X_{0} \cup X_{1}, \quad X_{1} \simeq[0,1)_{x} \times(\partial X)_{y}, \\
x: X \rightarrow[0,1), \quad y: X \rightarrow \partial X, \quad \text { real analytic on } X_{1},
\end{gathered}
$$


and, near the boundary,

$$
\widetilde{X} \simeq U \times \widetilde{\partial X}
$$

where $U$ is a neighbourhood of $[0,1)$ in $\mathbb{C}$ and $\widetilde{\partial X}$ is a Grauert tube of $\partial X$. The maps

$$
\widetilde{x}: \widetilde{X} \rightarrow U, \widetilde{y}: \widetilde{X} \rightarrow \widetilde{\partial X}
$$

are holomorphic.

We assume that $X$ is equipped with a scattering metric in the sense of Melrose [14], that is with a metric $g$ which near the boundary takes the form

$$
g=\left(\frac{d x}{x^{2}}\right)^{2}+\frac{h}{x^{2}}
$$

where $h \in \mathcal{C}^{\infty}\left(X, \operatorname{Sym}^{2} T^{*} X\right)$ restricts to a metric on $\partial X$. As discussed above, near the boundary we can use coordinates $(x, y), x \in U \subset \mathbb{C}$, a neighbourhood of 0 and $y \in \widetilde{\partial X}$, a Grauert tube of $\partial X$. We assume that

$$
h \text { is holomorphic in }\{(x, y):|\operatorname{Im} x| \leq \operatorname{Re} x / C, y \in \widetilde{\partial X}\} .
$$

A conic neighbourhood of this form is invariantly defined to first order, in the sense that a change of an analytic defining function changes the neighbourhood above into a set contained in, and containing, conic neighbourhoods defined using the new defining function. In Theorem 4 we additionally assume that the metric $g$ is holomorphic in a neighborhood of $X_{0}$ in $\tilde{X}$.

For finer upper bounds we need global assumptions related to the structure of the trapped set:

$$
\begin{gathered}
K \subset T^{*} X, K=\Gamma_{-} \cap \Gamma_{+}, \\
\Gamma_{ \pm}=\left\{m \in T^{*} X: x\left(\pi\left(\exp H_{g}(m)\right)\right) \nrightarrow 0 \text { as } t \longrightarrow \mp \infty\right\} .
\end{gathered}
$$

We now assume that the geodesic flow of $g$ is strictly hyperbolic on a neighbourhood of $K$. That can be guaranteed by making a stronger assumption that

$$
\text { all sectional curvatures of }\left(X^{\circ}, g\right) \text { are negative. }
$$


Because of the scattering structure of the metric they have to converge to 0 at the boundary.

Using (2.5) we can follow [23] and construct appropriate escape functions on neighbourhoods of $S^{*} X$. By an escape function, also known as Lyapunoff function, we mean $G \in \mathcal{C}^{1,1}\left(T^{*} X\right)$ such that

$$
H_{g} G \geq 0 .
$$

We start by observing that for any scattering manifold, there exists an escape function on a neighbourhood of the boundary:

$$
\begin{array}{r}
G_{0} \in \mathcal{C}^{\infty}\left(T^{*} X_{\epsilon}\right), \quad X_{\epsilon}=\{m \in X: 0<x(m)<\epsilon\}, \\
H_{g} G_{0}(m)>(2-\mathcal{O}(\epsilon)) g(m) .
\end{array}
$$

In fact, we can take $G_{0}=-\tau / x$, where $\eta=\tau d x / x+\mu \cdot d y / y$ is the first fundamental form. Expressing the Hamilton vector field in the coordinates $(x, y ; \tau, \mu), g=\tau^{2}+h(y, \mu)+x g^{\prime}$, we see, as in Section 3 of [16], that

$$
H_{g}\left(-\frac{\tau}{x}\right)=x^{\mathrm{sc}} H_{g}^{1,0}\left(-\frac{\tau}{x}\right)=2 \tau^{2}+2 h(y, \mu)=2 g+\mathcal{O}(x) g .
$$

This implies in particular that the trapped set intersected with the cosphere bundle, $K \cap S^{*} X$, is compact.

To construct an escape function near $K$, we recall that the flow is strongly hyperbolic near $K$ :

$$
\begin{gathered}
\exists W \subset S^{*} X, W^{\circ} \supset K \cap S^{*} X \exists C \forall m \in S^{*} X \exists E_{m}^{ \pm}, \\
E_{m}^{0} \subset T_{m}\left(S^{*} X\right), \operatorname{dim} E_{m}^{ \pm}=n-1, \operatorname{dim} E_{m}^{0}=1, \\
E_{m}^{+}+E_{m}^{-}+E_{m}^{0}=T_{m}\left(S^{*} X\right), \\
\quad d\left(\exp t H_{g}\right)_{m}\left(E_{m}^{\bullet}\right)=E_{\exp t H_{g}(m)}^{\bullet}, H_{g}(m) \in E_{m}^{0}, \\
\forall m \in W \quad\left\|d\left(\exp t H_{g}\right)_{m}\left(v_{ \pm}\right)\right\| \leq C \exp ( \pm C t)\left\|v_{ \pm}\right\|, v_{ \pm} \in E_{m}^{ \pm} .
\end{gathered}
$$

This holds globally (with constants uniform on compact sets) when we have (2.5). The assumptions of Section 5 of [23] are satisfied and, just as in Section 5 of [32], we can use them construct an escape function on a neighbourhood of the trapped set. More precisely, let

$$
S_{\delta}^{*} X=\left\{m \in T^{*} X: 1-\delta<g(m)<1+\delta\right\}, \quad K_{\delta}=K \cap S_{\delta}^{*} X .
$$


Then by the construction in Section 5 of [32] there exists $G_{1}$ defined on a neighbourhood $\Omega_{\epsilon}$ of $K_{2 \epsilon}$ in $S_{2 \epsilon}^{*} X$ such that

$$
\begin{gathered}
G_{1}, H_{g} G_{1} \in \mathcal{C}_{\mathrm{c}}^{1,1}\left(\Omega_{\epsilon} ; \mathbb{R}\right), G \in \mathcal{C}^{\infty}\left(\Omega_{\epsilon} \backslash K_{2 \epsilon}\right), \\
H_{g} G_{1} \geq\left\|\nabla G_{1}\right\|^{2} / C, H_{g} G_{1} \geq d\left(\bullet, K_{\epsilon}\right)^{2}
\end{gathered}
$$

on an open neighbourhood $W$ of $K_{\epsilon}$ in $S_{\epsilon}^{*} X \cap \Omega_{\epsilon}$,

$$
H_{g} G_{1} \geq 1 / C \text { on } \Omega_{\epsilon} \cap S_{\epsilon}^{*} X \backslash W \text {. }
$$

We can now apply the results of the appendix to [5] to conclude that the existence of $G_{0}$ and $G_{1}$ imply the existence of $G$ satisfying

$$
\begin{gathered}
G, H_{g} G \in \mathcal{C}_{\mathrm{c}}^{1,1}\left(S_{2 \epsilon}^{*} X ; \mathbb{R}\right), G \in \mathcal{C}^{\infty}\left(S_{2 \epsilon}^{*} X \backslash K_{2 \epsilon}\right), \\
H_{g} G \geq\|\nabla G\|^{2} / C, H_{g} G \geq d\left(\bullet, K_{\epsilon}\right)^{2} \\
\text { on an open neighbourhood } W \text { of } K_{\epsilon} \text { in } S_{\epsilon}^{*} X, \\
H_{g} G \geq 1 / C \text { on } S_{\epsilon}^{*} X \backslash W .
\end{gathered}
$$

A slightly modified version of this escape function will be crucial in the proof of Theorem 4.

When discussing Theorem 4 we will make the further assumption that

$$
g \text { is a real analytic metric on } X \text {. }
$$

Example. A simple example which satisfies the assumptions of Theorem 4 is given by the catenoid. Since in that case we can understand the asymptotic distribution of resonances in strips - they are generated by one hyperbolic orbit - it also shows that the result is optimal. Conceptually, it is the same example as the ones used to show the optimality of the results of [23] and [32]. Thus, we consider a manifold, $C$, with global coordinates $(r, \theta) \in \mathbb{R} \times \mathbb{S}^{1}$, and the metric $g=d r^{2}+\left(r^{2}+a^{2}\right) d \theta^{2}$. Taking $x=1 /|r|$ near infinity shows that $C$ satisfies the analytic assumptions. Since the mean curvature of $C \subset \mathbb{R}^{2}$ is 0 , the Gaussian curvature is negative. Hence the dynamical assumptions are also satisfied.

The Laplacian is given by

$$
\begin{aligned}
\Delta_{g} & =D_{r}^{2}+\frac{1}{i} \frac{r}{r^{2}+a^{2}} D_{r}+\frac{1}{r^{2}+a^{2}} D_{\theta}^{2} \\
& =\frac{1}{2 \pi} \sum_{n \in \mathbb{Z}}\left(D_{r}^{2}+\frac{1}{i} \frac{r}{r^{2}+a^{2}} D_{r}+\frac{1}{r^{2}+a^{2}} n^{2}\right) e^{i n\left(\theta-\theta^{\prime}\right)}
\end{aligned}
$$


and

$$
\begin{array}{r}
\left(r^{2}+a^{2}\right)^{\frac{1}{4}}\left(D_{r}^{2}+\frac{1}{i} \frac{r}{r^{2}+a^{2}} D_{r}+\frac{1}{r^{2}+a^{2}} n^{2}\right)\left(r^{2}+a^{2}\right)^{-\frac{1}{4}} \\
=D_{r}^{2}-\frac{3}{4} \frac{r^{2}}{\left(r^{2}+a^{2}\right)^{2}}+\frac{n^{2}+\frac{1}{2}}{r^{2}+a^{2}} .
\end{array}
$$

Considered as a semiclassical Schrödinger operator with $h=1 / n$, the last operator has an analytic potential with one non-degenerate maximum. Just as in [19], we can use the methods of Briet-Combes-Duclos and Sjöstrand to determine the asymptotic distribution of resonances in strips: for $|z|>C$ and $\operatorname{Im} z<C$ the resonances are asymptotically given by $z=\lambda^{2}$ with

$$
\begin{aligned}
& \operatorname{Re} \lambda=n a^{-1}+a_{0}+a_{1} n^{-1}+\cdots, \\
& \operatorname{Im} \lambda=a^{-1}\left(k+\frac{1}{2}\right)+b_{1}(k) n^{-1}+b_{2}(k) n^{-2}+\cdots, \\
& \quad k \in \mathbb{Z}, 0 \leq k
\end{aligned}
$$

For a numerical verification of this, see Figure 5 in Appendix B.

Many examples of real analytic surfaces with catenoidal ends and with complicated geodesic hyperbolic flows can be obtained from surfaces constructed in [12].

\section{Complex scaling on manifolds with boundary}

The purpose of this section is to present a generalized version of the complex scaling method. That method, which allows an effective meromorphic continuation of the resolvent, has a long tradition in mathematical physics, following the original work of Aguilar-Combes and Balslev-Combes. See for instance [9] for a historical account. Here we follow the approach of Sjöstrand and the second author [27], partly motivated by the approach of Helffer and Sjöstrand [8].

Let $P$ be a differential operator on $X$ with coefficients holomorphic in a set of the form (2.3). Let us denote the corresponding differential operator with holomorphic coefficients, $\widetilde{P}$. It is defined in a conic neighbourhood, $V$, of $\partial X$ in $\widetilde{X}$.

If $\Gamma \subset V \subset \widetilde{X}$ is a totally real submanifold of maximal dimension then $\widetilde{P}$ defines a differential operator on $\Gamma$ by

$$
P_{\Gamma} u=(\widetilde{P} \widetilde{u}) \Gamma_{\Gamma}
$$


where $u \in \mathcal{C}^{\infty}(\Gamma), \widetilde{u} \in \mathcal{C}^{\infty}(\widetilde{X})$,

$$
\left.\widetilde{u}\right|_{\Gamma}=u,\left.\quad \bar{\partial} \widetilde{u}\right|_{\Gamma}=\mathcal{O}_{N}\left(d(\bullet, \Gamma)^{N}\right), \text { for all } N,
$$

that is, $\widetilde{u}$ is an almost analytic extension from $\Gamma$ to $\widetilde{X}$.

The differential operator we shall consider is the Laplacian for the scattering metric satisfying analyticity assumptions near $\partial X$. In the notation of [14] we have

$$
\Delta \in \Psi_{\mathrm{sc}}^{2,0}(X)
$$

We also note that $\Delta-z \in \Psi_{\mathrm{sc}}^{2,0}(X)$ is elliptic as a scattering pseudodifferential operator for $z \in \mathbb{C} \backslash \overline{\mathbb{R}}_{+}$. In particular, it is Fredholm there, as the elements of the residual class $\Psi_{\mathrm{sc}}^{-\infty, \infty}(X)$ are compact by Proposition A.13.

We want to deform $X$ in $V \subset \widetilde{X}$ so that the operator induced by $\widetilde{\Delta}-z$ on the deformation is elliptic for $z$ in a conic neighbourhood of $\mathbb{R}$. Thus we define a family of totally real submanifolds

$$
X_{\theta} \subset \tilde{X}, \quad 0 \leq \theta<\theta_{0}
$$

as follows: Given $\epsilon>0, t_{1}>t_{0}>0$, there exists a sufficiently small $\theta$ and a deformation $\gamma_{\theta}$ of $[0,1)$ in $U$ satisfying (we write $[0,1) \ni t \mapsto \gamma_{\theta}(t)$ )

$$
\begin{gathered}
\gamma_{\theta}^{\prime}(t) \equiv e^{i \theta} \quad \text { for } t<t_{0} \\
\gamma_{\theta}(t) \equiv t \quad \text { for } t>t_{1}, \quad \gamma_{\theta}(0)=0 \\
\arg \gamma_{\theta}(t) \geq 0 \\
0 \leq \arg \gamma_{\theta}(t)-\arg \gamma_{\theta}^{\prime}(t) \leq \epsilon \\
0 \leq 2 \arg \gamma_{\theta}(t)-\arg \gamma_{\theta}^{\prime}(t) \leq \theta+\epsilon,
\end{gathered}
$$

(see Figure 1). For example, we can set

$$
\gamma_{\theta}(t)=e^{i \theta} t \phi(t)+t(1-\phi(t))
$$

where $\phi$ is a nonincreasing cutoff function that equals 1 for $t<t_{0}$ and 0 for $t>t_{1}$ : all except the last two properties in (3.1) are trivially satisfied. Since we have $\gamma_{\theta}(t)=t+i t \theta \phi+\mathcal{O}\left(\theta^{2}\right) \phi$ and $\gamma_{\theta}^{\prime}(t)=t^{-1} \gamma_{\theta}+$ $i t \theta \phi^{\prime}+\mathcal{O}\left(\theta^{2}\right) t \phi^{\prime}$, we compute $\gamma_{\theta}^{\prime}(t) / \gamma_{\theta}(t)=t^{-1}+i \theta \phi^{\prime}(t)+\mathcal{O}\left(\theta^{2}\right) \phi^{\prime}$, hence the penultimate inequality in (3.1) holds. Similarly,

$$
\gamma_{\theta}^{\prime}(t) / \gamma_{\theta}(t)^{2}=t^{-2}\left(1-i \theta \phi+i t \theta \phi^{\prime}+\mathcal{O}\left(\theta^{2}\right) \phi+\mathcal{O}\left(\theta^{2}\right) t \phi^{\prime}\right),
$$




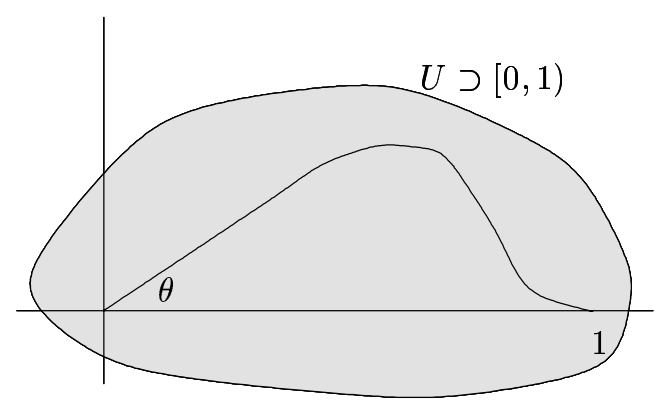

Figure 1: The contour $\gamma_{\theta}$ in $U$.

so the last property is also satisfied.

Using (2.2), and for $t_{1}$ and $\theta$ small enough, we then put $X_{\theta} \simeq$ $\left(\gamma_{\theta} \times \partial X\right) \cup X_{0}$, a totally real submanifold of $\widetilde{X}$. By the procedure described above, this gives

$$
\Delta_{\theta} \in \Psi_{\mathrm{sc}}^{2,0}\left(X_{\theta}\right)
$$

Here we use the space of scattering pseudodifferential operators: for a discussion of the scattering calculus and scattering Sobolev spaces, see Appendix A or [14].

The assumptions above guarantee that $\Delta_{\theta}-z$ is elliptic for $\arg z<$ $2 \theta$, or more precisely,

$$
\Delta_{\theta}-z \in \Psi_{\mathrm{sc}}^{2,0}\left(X_{\theta}\right) \text { is elliptic for } z \in \mathbb{C} \backslash e^{2 i \theta} \overline{\mathbb{R}}_{+} .
$$

In fact, at the boundary (i.e., in $\left.{ }^{\mathrm{sc}} \bar{T}_{\partial X_{\theta}}^{*} X_{\theta}\right), \Delta_{\theta}$ has the same the scattering principal symbol as $\left(x^{2} D_{x}\right)^{2}+x^{2} \Delta_{\partial X}, x \in \gamma_{\theta}$ :

$$
\left(\left(\gamma_{\theta}(t) / t\right)^{2}\left(\gamma_{\theta}^{\prime}(t)\right)^{-1} t^{2} D_{t}\right)^{2}+\left(\gamma_{\theta}(t) / t\right)^{2} t^{2} \Delta_{\partial X}, \quad 0<t<t_{1},
$$

and the conditions (3.1), with $\theta$ and $t_{1}$ small enough guarantee the ellipticity.

This immediately shows that

$$
\Delta_{\theta}-z: H_{\mathrm{sc}}^{2,0}\left(X_{\theta}\right) \longrightarrow L_{\mathrm{sc}}^{2}\left(X_{\theta}\right)
$$

is a Fredholm operator for $z \in \mathbb{C} \backslash e^{2 i \theta} \overline{\mathbb{R}}_{+}$, and consequently $\Delta_{\theta}$ has a discrete spectrum there. 
We now want to show that for some $\theta_{0}$

$$
\begin{aligned}
\sigma\left(\Delta_{\theta_{1}}\right) & \cap\left\{z: 2\left(\theta_{1}-\pi\right)<\arg z<2 \theta_{2}\right\} \\
= & \sigma\left(\Delta_{\theta_{2}}\right) \cap\left\{z: 2\left(\theta_{1}-\pi\right)<\arg z<2 \theta_{2}\right\}, \quad \theta_{2}<\theta_{1} \leq \theta_{0} .
\end{aligned}
$$

To prove (3.2) we start by recalling a well known local result about analytic continuation (see [27],[26] and references given there; Figure 2 illustrates the geometry):

Lemma 3.1. Let $\Omega \subset \mathbb{C}^{n}$ be open, $K \Subset \Omega$, and let $\Gamma_{t}, t \in[0,1]$ be a smoothly varying family of totally real submanifolds in $\Omega$, of maximal dimensions, such that $\Gamma_{t} \cap(\Omega \backslash K)=\Gamma_{t^{\prime}} \cap(\Omega \backslash K)$ for all $t, t^{\prime} \in[0,1]$. If $\widetilde{P}$ is a differential operator with holomorphic coefficients in $\Omega$ and $P_{\Gamma_{t}}$ is elliptic for each $t \in[0,1]$ then

$$
\begin{aligned}
& \left.\begin{array}{l}
u \in \mathcal{D}^{\prime}\left(\Gamma_{0}\right) \\
P_{\Gamma_{0}} u \text { extends holomorphically } \\
\text { to a neighbourhood of } \bigcup_{t \in[0,1]} \Gamma_{t}
\end{array}\right\} \\
& \Longrightarrow\left\{\begin{array}{l}
\forall t \in[0,1] \exists u_{t} \text { holomorphic near } \Gamma_{t}, \\
u_{t}=u_{s} \text { near } \Gamma_{s} \text { when }|s-t| \text { is small enough }
\end{array}\right.
\end{aligned}
$$

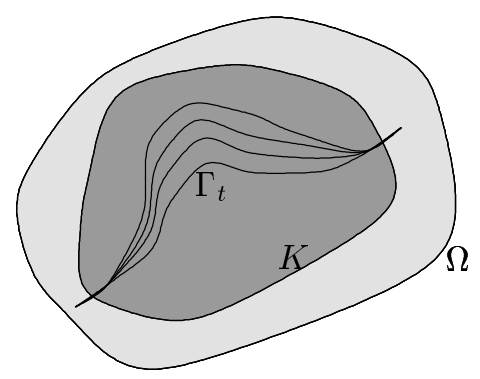

Figure 2: The family of totally real submanifolds $\Gamma_{t}$.

In particular $u$ extends holomorphically, to a a possibly multi-valued function, on a neighbourhood of $\bigcup_{t \in[0,1]} \Gamma_{t}$. We use this lemma to obtain the crucial

Lemma 3.2. Assume that $0 \leq \theta_{2}<\theta_{1}<\theta_{0}$ and that

$$
z \in \mathbb{C} \backslash \bigcup_{\theta_{2} \leq \theta \leq \theta_{1}} e^{i \theta} \overline{\mathbb{R}}_{+} .
$$


Then for all $k \in \mathbb{N}$

$$
\operatorname{dim} \operatorname{ker}\left(\Delta_{\theta_{1}}-z\right)^{k}=\operatorname{dim} \operatorname{ker}\left(\Delta_{\theta_{2}}-z\right)^{k} .
$$

Proof. For $\epsilon>0$ we introduce a new deformation $X_{\theta_{1}, \theta_{2}}^{\epsilon}$, obtained by replacing $\gamma_{\theta_{1}}$ by $\gamma_{\theta_{1}, \theta_{2}}^{\epsilon}$ shown in Figure 3:

$$
\begin{gathered}
\gamma_{\theta_{1}, \theta_{2}}^{\epsilon}=\gamma_{\theta_{1}}+\chi\left(\frac{x}{\epsilon}\right) \gamma_{\theta_{2}}, \quad \chi \in \mathcal{C}^{\infty}(\mathbb{R}), \\
\chi(t)=0 \text { for } t<1, \chi(t)=1 \text { for } t>2 .
\end{gathered}
$$

This defines a family of totally real submanifolds $X_{\theta_{1}, \theta_{2}}^{\epsilon}$, with the property that

$$
\left.\Delta_{\theta_{1}, \theta_{2}}^{\epsilon} \stackrel{\text { def }}{=} \Delta\right\rceil_{X_{\theta_{1}, \theta_{2}}^{\epsilon}} \in \Psi_{\mathrm{sc}}^{2,0}\left(X_{\theta_{1}, \theta_{2}}^{\epsilon}\right)
$$

is uniformly elliptic for $z \in \mathbb{C} \backslash \bigcup_{\theta_{2} \leq \theta \leq \theta_{1}} e^{i \theta} \overline{\mathbb{R}}_{+}$with all the symbol estimates independent of $\epsilon$. A local application of Lemma 3.1 (using a partition of unity on $\partial X$ and a construction of suitable $\Gamma_{t}$ 's covering $\left.X_{\theta_{1}, \theta_{2}}^{\epsilon}\right)$ shows that if $\left(\Delta_{\theta_{1}}-z\right)^{k} u_{\theta_{1}}=0$ then there exist an open set $W_{\theta_{1}, \theta_{2}} \subset \widetilde{X}$ such that

$$
\bigcup_{\theta_{2} \leq \theta \leq \theta_{1}}\left(X_{\theta} \backslash \partial X\right) \subset W_{\theta_{1}, \theta_{2}}
$$

and a (possibly multivalued) holomorphic function, $U$, on $W_{\theta_{1}, \theta_{2}}$, such that

$$
U\left\lceil X_{\theta_{1}}=u_{\theta_{1}} .\right.
$$

For $u_{\theta_{2}}=U\left\lceil X_{\theta_{2}}\right.$, we clearly have $\left(\Delta_{\theta_{2}}-z\right)^{k} u_{\theta_{2}}=0$, and we need to show that

$$
u_{\theta_{1}} \in L_{\mathrm{sc}}^{2}\left(X_{\theta_{1}}\right) \Longrightarrow u_{\theta_{2}} \in L_{\mathrm{sc}}^{2}\left(X_{\theta_{2}}\right) \text {. }
$$

Since $\left(\Delta_{\theta_{1}, \theta_{2}}^{\epsilon}-z\right)$ is uniformly elliptic in $\epsilon$, we obtain a parametrix:

$$
P_{\theta_{1}, \theta_{2}}^{\epsilon}\left(\Delta_{\theta_{1}, \theta_{2}}^{\epsilon}-z\right)^{k}=\mathrm{Id}+R_{\theta_{1}, \theta_{2}}^{\epsilon},
$$

with

$$
\begin{array}{r}
R_{\theta_{1}, \theta_{2}}^{\epsilon}=\mathcal{O}_{l, m}(1): H_{\mathrm{sc}}^{-m,-l}\left(X_{\theta_{1}, \theta_{2}}^{\epsilon}\right) \longrightarrow H_{\mathrm{sc}}^{m, l}\left(X_{\theta_{1}, \theta_{2}}^{\epsilon}\right), \\
P_{\theta_{1}, \theta_{2}}^{\epsilon}: H_{\mathrm{sc}}^{m, l}\left(X_{\theta_{1}, \theta_{2}}^{\epsilon}\right) \longrightarrow H_{\mathrm{sc}}^{m+2, l}\left(X_{\theta_{1}, \theta_{2}}^{\epsilon}\right) .
\end{array}
$$


Let $\psi \in \mathcal{C}_{\mathrm{c}}^{\infty}$ have the property that $\psi \equiv 1$ on supp $\chi$ and $\psi \equiv 0$ in a neighbourhood of 0 . Then

$$
u_{\theta_{1}, \theta_{2}}^{\epsilon}=U\left\lceil_{X_{\theta_{1}, \theta_{2}}^{\epsilon}}, \quad\left(\Delta_{\theta_{1}, \theta_{2}}^{\epsilon}-z\right)^{k} u_{\theta_{1}, \theta_{2}}^{\epsilon}=0,\right.
$$

and (for simplicity we put $k=1$ here)

$$
\begin{aligned}
\psi\left(\frac{x}{\epsilon}\right) u_{\theta_{1}, \theta_{2}}^{\epsilon} & =P_{\theta_{1}, \theta_{2}}^{\epsilon}\left(\left[\Delta_{\theta_{1}, \theta_{2}}^{\epsilon}, \psi(x / \epsilon)\right] u_{\theta_{1}, \theta_{2}}^{\epsilon}\right)-R_{\theta_{1}, \theta_{2}}^{\epsilon}\left(\psi(x / \epsilon) u_{\theta_{1}, \theta_{2}}^{\epsilon}\right) \\
& =P_{\theta_{1}, \theta_{2}}^{\epsilon}\left(\left[\Delta_{\theta_{1}}, \psi(x / \epsilon)\right] u_{\theta_{1}}\right)-R_{\theta_{1}, \theta_{2}}^{\epsilon}\left(\psi(x / \epsilon) u_{\theta_{1}, \theta_{2}}^{\epsilon}\right) .
\end{aligned}
$$

Hence,

$$
\begin{aligned}
\left\|\psi(x / \epsilon) u_{\theta_{1}, \theta_{2}}^{\epsilon}\right\|_{L_{\mathrm{sc}}^{2}\left(X_{\theta_{1}, \theta_{2}}^{\epsilon}\right) \leq} & C\left\|u_{\theta_{1}}\right\|_{L_{\mathrm{sc}}^{2}\left(X_{\theta_{1}}\right)}+C\left\|\psi(x / \epsilon) u_{\theta_{1}, \theta_{2}}^{\epsilon}\right\|_{H_{\mathrm{sc}}^{0, l}\left(X_{\theta_{1}, \theta_{2}}^{\epsilon}\right)} \leq C\left\|u_{\theta_{1}}\right\|_{L_{\mathrm{sc}}^{2}\left(X_{\theta_{1}}\right)}+C\left\|\psi(x / \delta) u_{\theta_{2}}\right\|_{H_{\mathrm{sc}}^{0, l}\left(X_{\theta_{2}}\right)} \\
& +C \delta^{l}\|\psi(x / \epsilon) u\|_{L_{\mathrm{sc}}^{2}\left(X_{\theta_{1}, \theta_{2}}^{\epsilon}\right)}
\end{aligned}
$$

$\delta>\epsilon$, since for $v$ supported where $x<\delta,\|v\|_{H_{\mathrm{sc}}^{0, l}}=\left\|x^{l} v\right\|_{L_{\mathrm{sc}}^{2}} \leq$ $\delta^{l}\|v\|_{L_{\mathrm{sc}}^{2}}$. The second term of the right hand side above is bounded, and consequently, by taking $\delta>\epsilon$ small, we obtain a uniform bound on $\left\|\psi(x / \epsilon) u_{\theta_{1}, \theta_{2}}^{\epsilon}\right\|_{L_{\mathrm{sc}}^{2}\left(X_{\theta_{1}, \theta_{2}}^{\epsilon}\right)}$, which in turn bounds $\left\|\widetilde{\psi}(x / \epsilon) u_{\theta_{2}}\right\|_{L_{\mathrm{sc}}^{2}\left(X_{\theta_{2}}\right)}$ for any $\widetilde{\psi} \in \mathcal{C}^{\infty}(\mathbb{R})$ such that $\psi \equiv 1$ on the support of $\widetilde{\psi}$. A uniform bound on $\left\|u_{\theta_{2}}\right\|_{L_{\mathrm{sc}}^{2}\left(X_{\theta_{2}}\right)}$ is an immediate consequence.

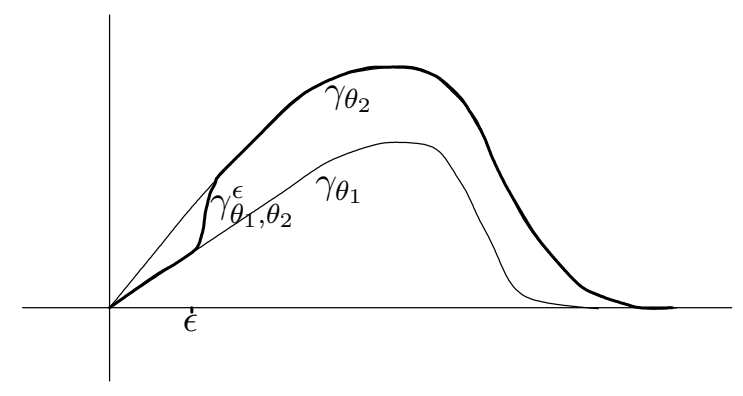

Figure 3: The interpolating contour $\gamma_{\theta_{1}, \theta_{2}}^{\epsilon}$.

In the case the kernel of $\left(\Delta_{\theta_{1}}-z\right)^{k}$ has higher dimension, the $u_{\theta_{2}}$ 's corresponding to independent $u_{\theta_{1}}$ 's are clearly independent. The rôles of $\theta_{1}$ and $\theta_{2}$ in the argument can be reversed and that concludes the proof. q.e.d.

The fact that considering kernels is sufficient is due to the following 
Lemma 3.3. For $z \in \mathbb{C} \backslash e^{2 i \theta} \overline{\mathbb{R}}_{+}$,

$$
\Delta_{\theta}-z: H_{s c}^{2,0}\left(X_{\theta}\right) \longrightarrow L_{s c}^{2}\left(X_{\theta}\right)
$$

is a Fredholm operator with index zero.

Proof. We already pointed out that the Fredholm property comes directly from the scattering calculus. To see that the index is 0 , we follow [27] and note that the index of $\Delta_{\theta}-z$ is constant in $\{(\theta, z): 0 \leq$ $\left.\theta<\theta_{0}, z \in \mathbb{C} \backslash e^{2 i \theta} \overline{\mathbb{R}}_{+}\right\}$. Thus, we can deform $\Delta_{\theta}-z$, first to $\Delta_{\theta}+i$ and then to $\Delta+i$ which is invertible. q.e.d.

As in [27], we now conclude that for $0 \leq \arg z<2 \theta$,

$$
\begin{gathered}
m_{X}(z)=\frac{1}{2 \pi i} \operatorname{tr} \int_{\gamma}\left(\widetilde{z}-\Delta_{\theta}\right)^{-1} d \widetilde{z}, \\
{[0,2 \pi) \ni t \mapsto \gamma(t)=z+\epsilon e^{i t}, \quad 0<\epsilon \ll 1,}
\end{gathered}
$$

gives a divisor independent of the choice of $\theta$ and of the choice of the contour $\gamma_{\theta}$ satifying (3.1). It remains to prove Theorem 1 and to show that $m_{X}(z)$ agrees with the one given there:

Proof of Theorem 1. Suppose that $z$ is not an eigenvalue of $\Delta_{\theta}$ for $\arg z<2 \theta$ and that $f \in \mathcal{C}_{\mathrm{c}}^{\infty}\left(X^{\circ}\right)$. We want to construct $R(z) f \in \mathcal{C}^{\infty}\left(X^{\circ}\right)$, which will be the continuation of $(P-\zeta)^{-1} f, \operatorname{Im} \zeta<0$. Let supp $f \subset K \Subset X^{\circ}$. We can choose $\gamma_{\theta}$ satisfying (3.1) so that no deformation takes place in $K$. We then put

$$
R(z) f=U \uparrow_{X_{0}}, \quad X_{0}=X, \quad\left(\Delta_{\theta}-z\right) u_{\theta}=f,
$$

where $U$ is the holomorphic continuation of $u_{\theta}$, as discussed in the proof of Lemma 3.2. Near a pole, $z_{0}$, the resolvent $\left(\Delta_{\theta}-z\right)^{-1}$, can written as

$$
\sum_{j=1}^{M\left(z_{0}\right)} \frac{A_{j}^{\theta}\left(z_{0}\right)}{\left(z-z_{0}\right)^{j}}+\widetilde{R}_{\theta}\left(z, z_{0}\right),
$$

where $A_{j}^{\theta}\left(z_{0}\right)$ 's are finite rank operators and $\widetilde{R}_{\theta}\left(z, z_{0}\right)$ is holomorphic near $z_{0}$. The first term, $A_{1}^{\theta}\left(z_{0}\right)$, is a projection onto the kernel of $\left(\Delta_{\theta}-z_{0}\right)^{M\left(z_{0}\right)}$ and its Schwartz kernel extends holomorphically to a neighbourhood of $\Gamma_{\widetilde{\theta}}, \widetilde{\theta}<\theta$, by the method described in Lemmas 3.1 and 3.2. The same is true for the coefficients of the more singular terms which are given by $A_{j}^{\theta}\left(z_{0}\right)=\left(\Delta_{\theta}-z_{0}\right)^{j-1} A_{1}^{\theta}\left(z_{0}\right)$. Unique continuation 
of solutions of $\left(\Delta_{\theta}-z\right)^{M\left(z_{0}\right)}$ (which, near $\partial X_{\theta}=\partial X$ is a restriction of a holomorphic operator to $\left.X_{\theta}\right)$ also shows that the rank of $A_{1}^{\theta}\left(z_{0}\right)$ is the same as the rank of $\mathbf{1}_{W} A_{1}^{\theta}\left(z_{0}\right) \mathbf{1}_{W}$, for any $W \Subset X^{\circ}$ with $X \backslash W$ sufficiently close to the boundary. Hence (3.4) provides a meromorphic continuation of $(\Delta-z)^{-1}: \mathcal{C}_{\mathrm{c}}^{\infty}\left(X^{\circ}\right) \rightarrow \mathcal{C}^{\infty}\left(X^{\circ}\right)$, with poles of finite rank and with the rank of the residues given by $m_{X}$. q.e.d.

We conclude with another characterization of multiplicities:

Proposition 3.4. Let $\arg z<2 \theta$ and $m_{X}(z)$ be given by (3.3). Then there exists a holomorphic family $\widetilde{R}(\widetilde{z})$ defined near $z$, and finite rank operators $A_{j}(z)$, such that

$$
R(\widetilde{z})=\sum_{j=1}^{M(z)} \frac{A_{j}(z)}{(\widetilde{z}-z)^{j}}+\widetilde{R}(\widetilde{z}),
$$

and if we define $V(z) \subset \mathcal{C}^{\infty}\left(X^{\circ}\right)$ by

$$
V(z)=\sum_{j=1}^{M(z)} A_{j}(z)\left(\mathcal{C}_{\mathrm{c}}^{\infty}\left(X^{\circ}\right)\right)
$$

then

$$
m_{X}(z)=\operatorname{dim} V(z)
$$

Proof. This follows from the usual structure corresponding to the Jordan normal form (see [27]) and already alluded to in the proof of Theorem 1 . The operators obtained by holomorphic continuation of the Schwartz kernels of $A_{j}(z): \mathcal{C}_{\mathrm{c}}^{\infty}\left(X^{\circ}\right) \rightarrow \mathcal{C}^{\infty}\left(X^{\circ}\right)$, have that structure and that proves the equivalence of the two notions of multiplicity: one given by the dimension of $V(z)$ and the other by the rank of the residue of $R(z)$. q.e.d.

\section{The FBI transformation and microlocal deformations}

To define a global FBI transformation on $X_{\theta}$ we first have to consider admissible phase functions defined on $T^{*} X_{\theta} \times X_{\theta}$. Since $X_{\theta}$ is not real analytic we will want functions which are holomorphic in a neighbourhood of $X_{\theta} \backslash W$, where $W \Subset X \cap X_{\theta}$. We allow the presence

of $W$ in case we only have the assumptions of Theorem 2. When the assumptions of Theorem 4 hold, then $W=\emptyset$. 
Let $D$ be a small conic neighbourhood of

$$
\Delta_{0}^{*}=\left\{(\alpha, z) \in T^{*} X^{\circ} \times X^{\circ}: \pi(\alpha)=z\right\}
$$

and let $\widetilde{D}$ be a small open neighbourhood of $\Delta_{0}^{*}$ in

$$
\left(T^{*} \widetilde{X^{\circ} \backslash W} \times \widetilde{X^{\circ} \backslash W}\right) \cup\left(T^{*} W_{1} \times W_{1}\right), \quad W \Subset W_{1}^{\circ}, \quad W_{1} \Subset X_{\theta} \cap X .
$$

Here $\sim$ denotes a Grauert tube. By a holomorphic function on $\widetilde{D}$ we will mean, somewhat abusively, a function which is holomorphic on $\widetilde{D} \cap\left(T^{*} \widetilde{X^{\circ} \backslash W} \times \widetilde{X^{\circ} \backslash W}\right)$ and $\mathcal{C}^{\infty}$ on $\widetilde{D} \cap\left(T^{*} W_{1} \times W_{1}\right)$.

A holomorphic function, $\widetilde{\phi}$, on $\widetilde{D}$, will be called an admissible phase function if, for $\alpha \in T^{*} X^{\circ}$ we have (with $\pi: T^{*} X \rightarrow X$, the natural projection)

$$
\begin{gathered}
\widetilde{\phi}(\alpha, \pi(\alpha))=0 \\
d_{z} \widetilde{\phi}(\alpha, z) \uparrow_{z=\pi(\alpha)}=-\alpha_{\xi} \\
d_{z}^{2}(\operatorname{Im} \widetilde{\phi}(\alpha, z)) \uparrow_{z=\pi(\alpha)} \gg \frac{1}{C}\left\langle\alpha_{\xi}\right\rangle \operatorname{Id}
\end{gathered}
$$

uniformly on compact sets,

$\phi$ is homogeneous of degree one in $\alpha_{\xi}$.

Note that for $\alpha \in T^{*} X^{\circ}$, that is for $\alpha$ real, $\operatorname{Im} d_{z} \widetilde{\phi} \uparrow_{z=\pi(\alpha)}=0$, so that $d_{z}^{2}(\operatorname{Im} \widetilde{\phi})$ is well defined.

By taking $\theta$ small enough, we can make $\widetilde{D}$ contain a neighbourhood of $T^{*} X_{\theta} \times X_{\theta}$. We can then define

$$
\phi_{\theta}=\widetilde{\phi} \uparrow_{T^{*} X_{\theta} \times X_{\theta}} .
$$

The properties of $\widetilde{\phi}(4.1)$, show that for

$$
\alpha \in\left(T^{*} X_{\theta} \subset T^{*} \widetilde{X^{\circ} \backslash W} \times \widehat{X^{\circ} \backslash W}\right) \cup\left(T^{*} W_{1} \times W_{1}\right),
$$

we have

$$
\begin{gathered}
\phi_{\theta}(\alpha, \pi(\alpha))=0 \\
d_{z} \phi_{\theta}(\alpha, z) \uparrow_{z=\pi(\alpha)}=-\alpha \\
\phi \text { is homogeneous of degree one in } \alpha_{\xi} .
\end{gathered}
$$

Slightly less obvious is the property that (4.3) $\left.d_{z}^{2}\left(\operatorname{Im} \phi_{\theta}(\alpha, z)\right)\right|_{z=\pi(\alpha)} \gg\left|\left\langle\alpha_{\xi}\right\rangle\right| \operatorname{Id}, \quad$ uniformly on compact sets. 
In fact, since $X_{\theta}$ is totally real, $d_{z}\left(\operatorname{Im} \phi_{\theta}(\alpha, z)\right) \uparrow_{z=\pi(\alpha)}=0$, and the Hessian is again well defined. Since $\theta$ is small, (4.3) follows from the assumption at $\theta=0$.

For a specific choice of $\widetilde{\phi}$ we can follow [24], [32] and put

$$
\widetilde{\phi}(\alpha, z)=-\left\langle\alpha_{\xi}, \exp _{\alpha_{x}}^{-1}(z)\right\rangle+\frac{i}{2}\left\langle\alpha_{\xi}\right\rangle \widetilde{d}\left(\alpha_{x}, z\right)^{2},
$$

where $\widetilde{d}^{2}$ is the holomorphic continuation of the square of the distance function.

For $u \in \mathcal{C}_{\mathrm{c}}^{\infty}\left(X_{\theta}^{\circ}\right)$, we can now define

$$
\begin{array}{r}
T u(\alpha ; h)=\int_{X_{\theta}} e^{i \phi_{\theta}(\alpha, z) / h} a_{\theta}(\alpha, z ; h) \chi_{\theta}\left(\alpha_{z}, z\right) u(z) d \operatorname{vol}_{g_{\theta}}, \\
\alpha=\left(\alpha_{z}, \alpha_{\zeta}\right) \in T^{*} X_{\theta}
\end{array}
$$

The amplitude $a_{\theta}$ is obtained by restricting an elliptic holomorphic symbol on $\widetilde{D}$, to $\widetilde{D} \cap\left(T^{*} X_{\theta} \times X_{\theta}\right)$ :

$$
a_{\theta}=a \uparrow_{\widetilde{D} \cap\left(T^{*} X_{\theta} \times X_{\theta}\right)}, \quad a \in S_{\mathrm{phg}}^{\frac{3 n}{4}, \frac{n}{4}}(\widetilde{D}) .
$$

Here $S_{\text {phg }}^{m, k}$ denotes the class of semiclassical polyhomogeneous holomorphic symbols bounded by $h^{-m}\left\langle\alpha_{\zeta}\right\rangle^{m}$ - see [24], (1.6)-(1.8). On $T^{*} W_{1} \times W_{1}$, they are assumed to be the usual symbols. The cutoff function $\chi_{\theta}$ is obtained by restricting a cutoff function on $\widetilde{X} \times \widetilde{X}$ to $X_{\theta} \times X_{\theta}$ :

$$
\chi_{\theta}=\chi\left\lceil_{X_{\theta} \times X_{\theta}}, \quad \chi \in \mathcal{C}^{\infty}(\widetilde{X} \times \widetilde{X}), \operatorname{supp} \chi \subset \pi(\widetilde{D})\right.
$$

where $\pi: \widetilde{T^{*} X} \times \widetilde{X} \rightarrow \widetilde{X} \times \widetilde{X}$. We assume that $\chi \equiv 1$ on a neighbourhood of $\left\{(\alpha, z) \in \widetilde{T^{*} X} \times \widetilde{X}: \pi(\alpha)=x\right\}$.

The transformation we introduced has all the essential properties of the global FBI transformation discussed in Section 1 of [24], when $W=\emptyset$ and all the properties of the $\mathcal{C}^{\infty}$ FBI transformation of [31]. Thus the same argument as those papers shows that there exists $b \in S^{\frac{3 n}{4}}, \frac{n}{4}(\widetilde{D})$ such that we have an approximate inverse of $T$ :

$$
S_{\theta} v(z)=\int_{T^{*} X_{\theta}} e^{-i \phi_{\theta}^{*}(\alpha, z)} b_{\theta}(\alpha, z, h) \chi_{\theta}\left(\alpha_{z}, z\right) d \alpha, \quad b_{\theta}=b \uparrow_{\widetilde{D} \cap T^{*} X_{\theta} \times X_{\theta}},
$$


where $\phi_{\theta}^{*}$ is the restriction to $T^{*} X_{\theta} \times X_{\theta}$ of the holomorphic continuation of $\overline{\widetilde{\phi}} \widetilde{D}_{\widetilde{D} \cap T^{*} X \times X}$ and $d \alpha$ is the canonical measure on $T^{*} X_{\theta}$. We have

$$
\begin{aligned}
& S_{\theta} T_{\theta} u=u+R_{\theta} u, \quad u \in L_{\text {comp }}^{2}\left(X_{\theta}\right), \\
& \begin{cases}\left|\partial_{z}^{k} \partial_{z^{\prime}}^{l} R_{\theta}\left(z, z^{\prime} ; h\right)\right| \leq C_{k, l, N} h^{N}, & W \neq \emptyset \\
\left|\partial_{z}^{k} \partial_{z^{\prime}}^{l} R_{\theta}\left(z, z^{\prime} ; h\right)\right| \leq C_{k, l} \exp (-1 / C h), & W=\emptyset,\end{cases}
\end{aligned}
$$

uniformly on compact sets. This follows as in [24] when $W=\emptyset$ and as in [31] when $W \neq \emptyset$, that is when we only use the $\mathcal{C}^{\infty} \mathrm{FBI}$ theory.

When $W=\emptyset$ we can use the holomorphy of the phase and the amplitude to deform the FBI transformation. Let $G_{\theta} \in \mathcal{C}_{\mathrm{c}}^{1,1}\left(T^{*} X_{\theta}\right)$. We can obtain a $\mathcal{C}^{1,1}$ extension of $G_{\theta}$ to the Grauert tube of $T^{*} X_{\theta}$ with the property that

$$
\left(d G_{\theta}\right)_{\rho} \bigwedge_{J T_{\rho}\left(T^{*} X_{\theta}\right)}=0
$$

where

$$
J: T_{\rho}\left(\widetilde{T^{*} X_{\theta}}\right)=T_{\rho}\left(\widetilde{T^{*} X}\right) \rightarrow T_{\rho}\left(\widetilde{T^{*} X}\right)=T_{\rho}\left(\widetilde{T^{*} X_{\theta}}\right)
$$

is the complex structure: Identify $T^{*} \widetilde{X_{\theta} \cap} U$, where $U$ is relatively compact neighbourhood of supp $G_{\theta}$, with a neighbourhood of the zero section in $T^{*}\left(T^{*} X_{\theta} \cap U\right)$ in which $J$ and the metric give the canonical symplectic structure; then make $G$ constant on the fibers.

Denoting the extension by the same symbol, $G \in \mathcal{C}^{1,1}\left(\widetilde{T^{*} X_{\theta}}\right)$, we now define a Lipschitz I-Lagrangian, $\mathbb{R}$-symplectic submanifold of $\widetilde{T^{*} X_{\theta}}$ :

$$
\Lambda_{t G_{\theta}}=\exp \left(t H_{G_{\theta}}^{\operatorname{Im}} \sigma\right)\left(T^{*} X_{\theta}\right) \subset \widetilde{T^{*} X_{\theta}},
$$

where

$$
\operatorname{Im} \sigma\left(v, H_{G_{\theta}}^{\operatorname{Im} \sigma}\right)=d G_{\theta}(v), \quad \sigma=d \alpha_{\zeta} \wedge d \alpha_{z} .
$$

Here by a Lipschitz manifold we mean a graph of a Lipschitz function. We remark that a Lipschitz graph in $\widehat{T^{*} X}$ over $T^{*} X$ (and hence for small $\theta$ over $T^{*} X_{\theta}$ ) which is I-Lagrangian (i.e., its almost everywhere defined tangent plane is Lagrangian with respect to $\operatorname{Im} \sigma$ ) can be locally written as a graph of a differential of a $\mathcal{C}^{1,1}$ function $\widetilde{G}$, on $T^{*} X \simeq T^{*} \mathbb{R}^{n}$ (locally):

$$
\begin{gathered}
\left\{(x, \xi) \in T^{*} \mathbb{C}^{n}: \operatorname{Im} x=\frac{\partial \widetilde{G}}{\partial \operatorname{Re} \xi}(\operatorname{Re} x, \operatorname{Re} \xi)\right. \\
\left.\operatorname{Im} \xi=-\frac{\partial \widetilde{G}}{\partial \operatorname{Re} x}(\operatorname{Re} x, \operatorname{Re} \xi)\right\} \\
(\operatorname{Re} x, \operatorname{Re} \xi) \in T^{*} \mathbb{R}^{n} \simeq T^{*} X
\end{gathered}
$$


see [24], [32] and the references given there.

The form - $\operatorname{Im} \zeta d z \Upsilon_{\Lambda_{t G_{\theta}}}$ is compactly supported and (formally) closed. Since $\Lambda_{t G_{\theta}}$ is close to $T^{*} X_{\theta}$,

$$
H_{c}^{1}\left(\Lambda_{t G_{\theta}}\right) \cong H_{c}^{1}\left(T^{*} X_{\theta}\right) \cong H^{2 n-1}\left(T^{*} X_{\theta}\right) \cong H^{2 n-1}\left(X_{\theta}\right)=0,
$$

where the second equivalence is by Poincaré duality. Hence $-\operatorname{Im} \zeta d z \uparrow_{\Lambda_{t G_{\theta}}}$ has a unique compactly supported primitive $H_{\Lambda_{t G_{\theta}}}$ which is a Lipschitz function on $\Lambda_{t G_{\theta}}$. More explicitly,

$$
H_{\Lambda_{t G_{\theta}}}=\int_{0}^{t} \exp \left((s-t) H_{G}^{\operatorname{Im} \sigma}\right)^{*}\left(G-\left\langle H_{G}^{\operatorname{Im} G}, \operatorname{Im}(\zeta d z)\right\rangle\right) d s
$$

(see Example in Section 1 of [24]). Clearly, $H_{\Lambda_{t G_{\theta}}} \in \operatorname{Lip}\left(\Lambda_{t G_{\theta}} ; \mathbb{R}\right)$. It is unique through the normalization that it is equal to 0 for $\left|\alpha_{\zeta}\right|$ large enough.

Thanks to the global properties of the amplitude and the phase function of $T_{\theta}$ we can continue it in $\alpha$ to a neighbourhood $\widetilde{T^{*} X_{\theta}}$ of $T^{*} X_{\theta}$ in $T^{*} \widetilde{X}$ and in particular we can define

$$
T_{\Lambda_{t G_{\theta}}} u(\alpha ; h)=T_{\theta} u \Upsilon_{\Lambda_{t G_{\theta}}}(\alpha ; h), \quad \alpha \in \Lambda_{t G_{\theta}} .
$$

A deformation argument which despite the irregularity of $\Lambda_{t G_{\theta}}$ proceeds as in Section 1 of [24] gives an approximate inverse, $S_{\Lambda_{t G_{\theta}}}$, with the same properties as $S_{\theta}$ above.

Following [24] (see also [32]) we can now put, for $u \in \mathcal{C}_{\mathrm{c}}^{\infty}\left(X_{\theta}\right)$,

$$
\begin{aligned}
& \|u\|_{H\left(X_{\theta} ; \Lambda_{t G_{\theta}},\left\langle\alpha_{\zeta}\right\rangle^{m}\right)}^{2} \\
& \quad=\int_{\Lambda_{t G_{\theta}}}\left|T_{\Lambda_{t G_{\theta}}} u(\alpha ; h)\right|^{2}\left|\left\langle\alpha_{\zeta}\right\rangle\right|^{2 m} e^{-2 H_{\Lambda_{t G_{\theta}}}(\alpha) / h} d \alpha,
\end{aligned}
$$

where again $d \alpha$ is the canonical measure on the totally real (as a submanifold of $T^{*} \widetilde{X}$ ), $\mathbb{R}$-symplectic manifold $\Lambda_{t G_{\theta}}$. Using $S_{\Lambda_{t G_{\theta}}}$ we can show the independence of this norm of the choice of a specific phase function (see Lemma 7.3 in [32] for a similar argument).

As in [32], we now want to replace the norm given in (4.6) by a locally-defined norm which in which we can do the Bergman kernel computations which will be necessary to prove Lemma 5.2.

We first introduce some local coordinates. Recall that $K$ denotes the trapped set of the geodesic flow. Let $Y$ be a neighbourhood of $\pi(K)$ 
in $X$ such that $\bar{Y} \Subset X \cap X_{\theta}$. We denote by $\widetilde{Y}$ a neighbourhood of $Y$ in $\widetilde{X}$. We then cover $\widetilde{Y}$ by holomorphic coordinate charts:

$$
\tilde{Y} \subset \bigcup_{q=1}^{Q} \Omega_{q}, \quad \chi_{q}: \Omega_{q} \rightarrow \mathbb{C}^{n}, \quad \chi_{q}: \Omega_{q} \cap X \rightarrow \mathbb{R}^{n}
$$

We also assume that there exist $\Omega_{q}^{\prime}, \widetilde{X} \supset \Omega_{q}^{\prime} \ni \Omega_{q}$, such that $\chi_{q}$ extend to holomorphic coordinate maps on the $\Omega_{q}^{\prime}$ 's.

We now recall the definition of the unitary Bargman type FBI transform: let

$$
\mathcal{L}(d z)=(2 i)^{-1} d \bar{z} \wedge d z \text { and } \Phi_{0}(z)=(\operatorname{Im} z)^{2} / 2
$$

and define

$$
\begin{gathered}
T_{0}: L^{2}\left(\mathbb{R}^{n}\right) \rightarrow H_{\Phi_{0}}^{2}\left(\mathbb{C}^{n}\right)=\left\{u \in \mathcal{D}^{\prime}\left(\mathbb{C}^{n}\right): \int_{\mathbb{C}^{n}}|u(z)|^{2} e^{-2 \Phi_{0}(z) / h} \mathcal{L}(d z)\right. \\
\left.u \text { is holomorphic on } \mathbb{C}^{n}\right\} \\
T_{0} u(z, h)=c_{n} h^{-\frac{3 n}{4}} \int_{\mathbb{R}^{2}} e^{-(x-z)^{2} / 2 h} u(x) d x
\end{gathered}
$$

Let $\Omega_{q}^{\sharp}$ be an open precompact subset of $\chi_{q}\left(\Omega_{q} \cap X\right)+i \mathbb{R}^{n} \subset \mathbb{C}^{n}$ which can be identified with a subset of $T_{\Omega_{q}}^{*} X$ using $\chi_{q}$.

We now define a new transformation $T_{q}$ by

$$
T_{q} u(z, h)=T_{0}\left(\left(\chi_{q}^{-1}\right)^{*}\left(u\left\lceil_{\Omega_{q}^{\prime} \cap X}\right)\right) \Gamma_{\Omega_{q}^{\sharp}},\right.
$$

which is independent of the extensions of $u\left\lceil_{\Omega_{q}^{\prime} \cap X}\right.$ up to exponentially small errors.

The main technical result of Section 6 of [32] relates the globallydefined norm (4.6) to norms defined using the local transforms (4.7). It is based on techniques of [8], [23] and we recall it without proof:

Proposition 4.1 We can choose $\Omega_{q}^{\sharp}$ in (4.7) and

$$
\begin{gathered}
\Phi_{t, q}(w)=\frac{1}{2}(\operatorname{Im} w)^{2}+F_{q, t}, \quad F_{q, t} \in \mathcal{C}^{1,1}\left(\Omega_{q}^{\sharp}\right), \\
\psi_{q} \in \mathcal{C}_{\mathrm{c}}^{\infty}\left(\Omega_{q}^{\sharp} ;[0,1]\right), \widetilde{\psi} \in \mathcal{C}_{\mathrm{c}}^{\infty}\left(T^{*} X_{\theta} ;[0,1]\right), \widetilde{\psi} \equiv 1 \text { on a neighbourhood of }
\end{gathered}
$$


$S_{Y}^{*} X_{\theta}$ in $\widetilde{T^{*} X}$, such that the norm

$$
\begin{aligned}
& \|\| u \|_{H\left(X_{\theta}, \Lambda_{t G_{\theta}} ;\left\langle\alpha_{\xi}\right\rangle^{m}\right)}^{2} \\
& =\sum_{q=1}^{Q} \int_{\Omega_{q}^{\sharp}} \psi_{q}\left|T_{q} u(w ; h)\right|^{2} e^{-2 \Phi_{q, t}(w) / h} \mathcal{L}(d w) \\
& \quad+\int_{\Lambda_{t G_{\theta}}}(1-\widetilde{\psi})\left|T_{\Lambda_{t G_{\theta}}} u(\alpha ; h)\right|^{2} e^{-2 H_{\Lambda_{t G_{\theta}}}(\alpha) / h}\left|\left\langle\alpha_{\xi}\right\rangle\right|^{2 m} d \alpha,
\end{aligned}
$$

and the corresponding inner product, are equivalent to the ones given by (4.6), uniformly on compact sets:

$$
\frac{1}{C}\|u\|_{H\left(X_{\theta}, \Lambda_{t G_{\theta}} ; 1\right)} \leq\|\| u\left\|_{H\left(X_{\theta}, \Lambda_{t G_{\theta}} ; 1\right)} \leq C\right\| u \|_{H\left(X_{\theta}, \Lambda_{t G_{\theta}} ; 1\right)},
$$

$u \in \mathcal{C}_{\mathrm{c}}^{\infty}(W), \bar{W} \Subset X_{\theta}, C=C(W)$.

The global norms we will now use are given by

$$
\begin{array}{ll}
\|\| u\|\|^{2}=\|\psi u\|_{H\left(X_{\theta} ; T^{*} X_{\theta}, 1\right)}^{2}+\|(1-\psi) u\|_{L_{\mathrm{sc}}^{2}\left(X_{\theta}\right)}^{2} & W \neq \emptyset \\
\|\| u\|\|_{t G_{\theta}}^{2}=\|\psi u\|_{H\left(X_{\theta}, \Lambda_{t G_{\theta}} ; 1\right)}^{2}+\|(1-\psi) u\|_{L_{\mathrm{sc}}^{2}\left(X_{\theta}\right)}^{2} & W=\emptyset
\end{array}
$$

where $\psi \in \mathcal{C}_{\mathrm{c}}^{\infty}\left(X_{\theta}\right), \psi \equiv 1$ on a neighbourhood of $X \cap X_{\theta}$ and $\left(\Delta_{\theta}-z\right)^{-1} \in \Psi_{\mathrm{sc}}^{2,0}\left(X_{\theta}\right)$ is elliptic (for $\arg z>-2 \theta$ ) on the support of $(1-\psi)$.

We note that these norms are equivalent to the usual $L_{\mathrm{sc}}^{2}$ norm (trivially so in the case where $W \neq \emptyset$ ) uniformly in $h$ for the first norm but without uniformity in $h$ for the second one:

$$
\begin{gathered}
C_{\psi}^{-1}\|u\|_{L_{\mathrm{sc}}^{2}\left(X_{\theta}\right)} \leq\|\| u\left\|^{2} \leq C_{\psi}\right\| u \|_{L_{\mathrm{sc}}^{2}\left(X_{\theta}\right)}, \\
e^{-C / h}\|u\|_{L_{\mathrm{sc}}^{2}\left(X_{\theta}\right)} \leq\|u\|_{t G_{\theta}}^{2} \leq e^{C / h}\|u\|_{L_{\mathrm{sc}}^{2}\left(X_{\theta}\right)}
\end{gathered}
$$

\section{Proof of the main estimate}

To prove Theorems 2 and 4 we will use the method originating from [23] with improvements from [27] and [28].

Proof of Theorem 2. We will show that for

$$
\omega_{0} \in \mathbb{C}, \quad \operatorname{Re} \omega_{0}>0, \quad \operatorname{Im} \omega_{0}=-r_{0}<0,
$$


we have

$$
\begin{aligned}
&\left\|\left(h^{2} \Delta_{\theta}-\omega_{0}\right) u\right\| \|^{2} \geq\left(\left(r_{0}+\mu\right)^{2}-\mathcal{O}(h)\right)\|\| u\|\|^{2} \\
&-\mathcal{O}\left(\mu+\epsilon_{0}\right)\left\|Q_{\mu, h} u\right\| \|^{2}, \\
& \operatorname{rank} Q_{\mu, \theta}=\mathcal{O}_{\theta, \mu}\left(h^{-n}\right), \quad h<h_{0}(\theta), \quad \mu<\mu_{0}(\theta)
\end{aligned}
$$

where $\epsilon_{0}>0$ is fixed by our construction of the deformation $\gamma_{\theta}$, and can be made as small as desired.

To obtain this let us introduce $\psi_{1} \in \mathcal{C}_{\mathrm{c}}^{\infty}\left(X_{\theta}^{\circ}\right)$ and $\psi_{2} \in \mathcal{C}^{\infty}\left(X_{\theta}\right)$, such that $\psi_{1}^{2}+\psi_{2}^{2} \equiv 1$. Then

$$
\begin{aligned}
\left\|\left(h^{2} \Delta_{\theta}-\omega_{0}\right) u \mid\right\|^{2}= & \left\|\left|\psi_{1}\left(h^{2} \Delta_{\theta}-\omega_{0}\right) u\right|\right\|^{2}+\left\|\mid \psi_{2}\left(h^{2} \Delta_{\theta}-\omega_{0}\right) u\right\| \|^{2} \\
\geq & \sum_{i=1,2}\left\|\left(h^{2} \Delta_{\theta}-\omega_{0}\right) \psi_{i} u\right\|\left\|^{2}-\sum_{i=1,2}\right\|\left[\psi_{i}, h^{2} \Delta_{\theta}\right] u\|\|^{2} \\
& -2 \sum_{i=1,2}\left\|\mid \psi_{i}\left(h^{2} \Delta_{\theta}-\omega_{0}\right) u\right\|\|\|\left[\psi_{i}, h^{2} \Delta_{\theta}\right] u \| .
\end{aligned}
$$

By Proposition A.10, $\left[\psi_{i}, h^{2} \Delta_{\theta}\right] \in \Psi_{\mathrm{sc}, h}^{1, \infty,-1}\left(X_{\theta}\right)=h \Psi_{\mathrm{sc}, h}^{1, \infty, 0}\left(X_{\theta}\right)$. Furthermore,

$$
\begin{aligned}
\|u\|_{H_{\mathrm{sc}, h}^{1, \infty}\left(X_{\theta}\right)}^{2} & \leq C\left(\left|\left\langle\left(h^{2} \Delta_{\theta}-\omega_{0}\right) u, u\right\rangle\right|+\|u\|_{L_{\mathrm{sc}}^{2}\left(X_{\theta}\right)}\right) \\
& \leq C\left(\|\| u\|+\| \mid\left\|\left(h^{2} \Delta_{\theta}-\omega_{0}\right) u\right\| \|\right)^{2} .
\end{aligned}
$$

Hence

$$
\left\|||\left[\psi_{i}, h^{2} \Delta_{\theta}\right] u\right\| \mid=\mathcal{O}(h)\left(\|\| u\|\mid+\|\left(h^{2} \Delta_{\theta}-\omega_{0}\right) u\|\|\right), \quad i=1,2,
$$

and we conclude that

$$
\begin{aligned}
\left\|\left(h^{2} \Delta_{\theta}-\omega_{0}\right) u\right\| \|^{2} \geq & \left.\sum_{i=1,2}\left\|\left(h^{2} \Delta_{\theta}-\omega_{0}\right) \psi_{i} u\right\|\right|^{2} \\
& -\mathcal{O}(h)\left(\|\|\left(h^{2} \Delta_{\theta}-\omega_{0}\right) u\left\|^{2}+\right\|\|u\|^{2}\right) .
\end{aligned}
$$

Thus we can consider $\psi_{i} u, i=1,2$, separately. For the case $i=2$ we need the following

Lemma 5.1. If supp $\psi_{2}$ is sufficiently close to $\partial X_{\theta}$ then

$$
\begin{gathered}
\left\|\mid\left(h^{2} \Delta_{\theta}-\operatorname{Re} \omega_{0}\right) \psi_{2} u\right\|\left\|\geq \frac{1}{C_{\theta, \psi}}\right\| \psi_{2} u \|, \\
\operatorname{Im}\left\langle\Delta_{\theta} \psi_{2} u, \psi_{2} u\right\rangle \geq-C \epsilon\left\|\psi_{2} u\right\|^{2},
\end{gathered}
$$

where $\epsilon=\max \left\{x(m): m \in \operatorname{supp} \psi_{2}\right\}, 0<\epsilon \ll \theta$. 
Proof. To prove the first inequality we extend $\Delta_{\theta}$ from supp $\psi_{2}$ to an operator $Q_{\theta} \in \Psi_{\mathrm{sc}, h}^{2,0,0}\left(X_{\theta}\right)$, so that $\sigma_{\mathrm{sc}}^{2,0,0}\left(Q_{\theta}\right)-w \neq 0$ for $w \in \mathbb{R}_{+}$. This is possible since it holds for $\sigma_{\mathrm{sc}}^{2,0,0}\left(\Delta_{\theta}\right)$ over the support of $\psi_{2}$ (which taken to be sufficiently close to $\left.\partial X_{\theta}\right)$. Then $h^{2} Q_{\theta}-\operatorname{Re} \omega_{0}$ is uniformly elliptic in the semiclassical scattering calculus (see Proposition A.11 in Appendix A), and consequently we have a parametrix:

$$
\begin{gathered}
T_{\theta}\left(h^{2} Q_{\theta}-\operatorname{Re} \omega_{0}\right)=\mathrm{Id}+R_{\theta}, \\
T_{\theta}=\mathcal{O}_{\theta}(1): L_{\mathrm{sc}}^{2}\left(X_{\theta}\right) \rightarrow H_{\mathrm{sc}, h}^{2}\left(X_{\theta}\right), \\
R_{\theta}=\mathcal{O}_{\theta}(h): L_{\mathrm{sc}, h}^{2}\left(X_{\theta}\right) \rightarrow L_{\mathrm{sc}}^{2}\left(X_{\theta}\right) .
\end{gathered}
$$

Hence for small $h,\left(I+R_{\theta}\right)^{-1}=\mathcal{O}_{\theta}(1)$ exists and

$$
\begin{aligned}
\left\|\mid\left(h^{2} \Delta_{\theta}-\operatorname{Re} \omega_{0}\right) \psi_{2} u\right\|^{2}= & \left\|\left(h^{2} Q_{\theta}-\operatorname{Re} \omega_{0}\right) \psi_{2} u\right\| \|^{2} \\
\geq & C_{\psi}^{-1}\left\|\left(h^{2} Q_{\theta}-\operatorname{Re} \omega_{0}\right) \psi_{2} u\right\|_{L_{\mathrm{sc}}^{2}\left(X_{\theta}\right)}^{2} \\
\geq & C(\theta)^{-1} C_{\psi}^{-1} \|\left(I+R_{\theta}\right)^{-1} \\
& T_{\theta}\left(h^{2} \Delta_{\theta}-\operatorname{Re} \omega_{0}\right) \psi_{2} u \|_{L_{\mathrm{sc}}^{2}\left(X_{\theta}\right)}^{2} \\
= & C(\theta)^{-1} C_{\psi}^{-1}\left\|\psi_{2} u\right\|_{L_{\mathrm{sc}}^{2}\left(X_{\theta}\right)}^{2} \\
\geq & C(\theta)^{-1} C_{\psi}^{-2}\left\|\psi_{2} u\right\| \|^{2} .
\end{aligned}
$$

To prove the second inequality we can assume that

$$
X_{\theta} \cap \operatorname{supp} \psi_{2} \simeq\left(e^{i \theta}[0, \epsilon] \times \partial X\right) \cap \operatorname{supp} \psi_{2},
$$

so that (see Section 3 above and Section 3 in [14]),

$$
\Delta_{\theta}=e^{2 i \theta}\left[\left(x^{2} D_{x}\right)^{2}+i(n-1) x^{3} D_{x}+x^{2} \Delta_{\partial X}\right]+x^{3} \operatorname{Diff}_{\mathrm{b}}^{2}(X),
$$

and consequently

$$
\begin{aligned}
& \operatorname{Im}\left\langle\Delta_{\theta} \psi_{2} u, \psi_{2} u\right\rangle \\
& \quad \geq \sin 2 \theta\left(\left\|x^{2} D_{x}\left(\psi_{2} u\right)\right\|_{L_{\mathrm{sc}}^{2}}+\left\langle x^{2} \Delta_{\partial X}\left(\psi_{2} u\right), \psi_{2} u\right\rangle\right) \\
& \quad-C \epsilon\left(\left\|x^{2} D_{x}\left(\psi_{2} u\right)\right\|_{L_{\mathrm{sc}}^{2}}+\left\langle x^{2} \Delta_{\partial X}\left(\psi_{2} u\right), \psi_{2} u\right\rangle+\left\|\psi_{2} u\right\|_{L_{\mathrm{sc}}^{2}}^{2}\right),
\end{aligned}
$$

from which the inequality follows. q.e.d.

Using the lemma we conclude that

$$
\begin{aligned}
\left\|\left|\left(h^{2} \Delta_{\theta}-\omega_{0}\right) \psi_{2} u \|\right|^{2}=\right. & \|\|\left(h^{2} \Delta_{\theta}-\operatorname{Re} \omega_{0}\right) \psi_{2} u\left\|\left.\right|^{2}+r_{0}^{2}\right\|\left\|\psi_{2} u\right\| \| \\
& +2 r_{0} \operatorname{Im}\left\langle h^{2} \Delta_{\theta} \psi_{2} u, \psi_{2} u\right\rangle \\
\geq & \left(C_{\theta, \psi}^{-2}+r_{0}^{2}-C \epsilon h^{2} r_{0}\right)\left\|\mid \psi_{2} u\right\| \|
\end{aligned}
$$


and hence we need to prove (5.1) with $u$ replaced by $\psi_{1} u$. There are many alternative ways to proceed and we choose the $\mathcal{C}^{\infty}$ FBI transform approach as described in [31] (see methods of Sections 3 and 5 of [28], and references given there for a slightly different approach). We first demand that $\psi \psi_{1}=\psi_{1}$, as we certainly may by changing $\psi$ in the definition of $\|\bullet\|$. Since that means changing $\psi$ in a way dependent on $\theta$, we obtain a new $\theta$ dependent constant. Then

$$
\begin{aligned}
\|\|\left(h^{2} \Delta_{\theta}-\omega_{0}\right)\left(\psi_{1} u\right)\|\|^{2} \\
\left.=\| T\left(h^{2} \Delta_{\theta}-\omega_{0}\right) \psi_{1} u\right) \|_{L^{2}\left(T^{*} X_{\theta}, d \alpha\right)}^{2} \\
=\left\|\left|\sigma_{2}\left(\Delta_{\theta}\right)-\omega_{0}\right| T\left(\psi_{1} u\right)\right\|_{L^{2}\left(T^{*} X_{\theta}, d \alpha\right)}^{2}+\mathcal{O}(h)\left\|\mid\langle h D\rangle \psi_{1} u\right\|^{2} \\
\geq \\
\quad\left(r_{0}+\mu\right)^{2}\left\|T\left(\psi_{1} u\right)\right\|_{L^{2}\left(T^{*} X_{\theta}, d \alpha\right)}^{2} \\
\quad-\mathcal{O}\left(\mu+\epsilon_{0}\right)\left\|\mathbf{1}_{\left\{\alpha:\left|\sigma_{2}\left(\Delta_{\theta}\right)-\omega_{0}\right| \leq r_{0}+\mu\right\}} T\left(\psi_{1} u\right)\right\|_{L^{2}\left(T^{*} X_{\theta}, d \alpha\right)}^{2} \\
\quad-\mathcal{O}(h)\left\|\left|\psi_{1} u\right|\right\|^{2},
\end{aligned}
$$

where we used the facts that $\operatorname{Im} \sigma_{2}\left(\Delta_{\theta}\right)>-\epsilon_{0} \operatorname{Re} \sigma_{2}\left(\Delta_{\theta}\right)$ with $\epsilon_{0}$ as small as desired depending on the construction of $\gamma_{\theta}$ (described in (3.1); see also the proof of Lemma 5.1), hence

$$
\left|\sigma_{2}\left(\Delta_{\theta}\right)-\omega_{0}\right| \leq r_{0}+\mu \Longrightarrow\left(r_{0}+\mu\right)^{2}-\left|\sigma_{2}\left(\Delta_{\theta}\right)-\omega_{0}\right|^{2} \leq C\left(\mu+\epsilon_{0}\right) .
$$

We have also used the intertwining property of $T$ : when $P$ is a second order semi-classical differential operator then

$$
\begin{aligned}
\left\|T P\left(\psi_{1} u\right)\right\|_{L^{2}\left(T^{*} X_{\theta}, d \alpha\right)}^{2}= & \left\|\sigma_{2}(P) T\left(\psi_{1} u\right)\right\|_{L^{2}\left(T^{*} X_{\theta}, d \alpha\right)}^{2} \\
& +\mathcal{O}(h)\|\langle h D\rangle u\|_{L^{2}\left(X_{\theta}\right)}^{2} .
\end{aligned}
$$

We were able to drop $\langle h D\rangle$ in the last inequality in (5.3) because of the classical ellipticity of $\Delta_{\theta}-\omega_{0}$. We are using $\sigma_{2}$ to denote the (total) semiclassical symbol of a second order operator on a compact manifold or over the interior of a noncompact one.

It is a well known fact (recalled in [31]) that there exists an operator $\widetilde{Q}_{\theta, \mu}$ such that

$$
\begin{array}{r}
\Pi 1_{\left\{\alpha:\left|\sigma_{2}\left(\Delta_{\theta}\right)-\omega_{0}\right| \leq r_{0}+\mu\right\}} \Pi=\widetilde{Q}_{\theta, \mu}+\mathcal{O}_{N}\left(h^{N}\left\langle\alpha_{\xi}\right\rangle^{-N}\right), \\
\operatorname{rank} \widetilde{Q}_{\theta, \mu}=\mathcal{O}\left(h^{-n}\right),
\end{array}
$$

where $\Pi$ is the orthogonal projection on $L^{2}\left(T^{*} X_{\theta}\right)$ onto the image of 
$T \widetilde{\psi}_{1}, \widetilde{\psi}_{1} \in \mathcal{C}_{\mathrm{c}}^{\infty}\left(X_{\theta}\right), \widetilde{\psi}_{1} \equiv 1$ on supp $\psi_{1}$. This concludes the proof of (5.1).

For the reader's convenience we now recall the counting argument from $[23,27,28]$. Let us take $\omega_{0}=1-i r_{0}$ with $r_{0}$ small and let $z_{1}, \cdots, z_{N}$ be the eigenvalues of $h^{2} \Delta_{\theta}-\omega_{0}$ in $D\left(\omega_{0}, r_{0}+\mu / 2\right)$. The characteristic values of $h^{2} \Delta_{\theta}-\omega_{0}$ are defined as the the eigenvalues $\mu_{1}, \mu_{2}, \ldots$ of the self-adjoint operator $\left[\left(h^{2} \Delta_{\theta}-\omega_{0}\right)^{*}\left(h^{2} \Delta_{\theta}-\omega_{0}\right)\right]^{\frac{1}{2}}$ (with the convention that if there are only finitely many eigenvalues we repeat the bottom of the essential spectrum infinitely many times). A version of the celebrated Weyl inequality says that

$$
\mu_{1} \cdots \mu_{N} \leq\left|z_{1}-\omega_{0}\right| \cdots\left|z_{N}-\omega_{0}\right|
$$

(see Appendix A to [23]). Thus we need to estimate the number of $\mu_{j}$. The estimate (5.1) and the max-min principle show that

$$
\begin{gathered}
M(\mu) \stackrel{\text { def }}{=} \#\left\{\mu_{j}: \mu_{j} \leq r_{0}+\mu\right\} \leq C_{\theta, \mu} h^{-n}, \\
\mu<\inf \sigma_{\text {ess }}\left(\left[\left(h^{2} \Delta_{\theta}-\omega_{0}\right)^{*}\left(h^{2} \Delta_{\theta}-\omega_{0}\right)\right]^{\frac{1}{2}}\right)-r_{0},
\end{gathered}
$$

where, by Lemma 5.1, inf $\sigma_{\text {ess }}>r_{0}+1 / C_{\theta}-$ see (5.2). (Equation (5.2) suffices because the essential spectrum is determined by behaviour near infinity - see for instance [2].) Equation (5.1) also shows that $\mu_{1}>r_{0}-\epsilon$ where $\epsilon>0$ can be made as small as desired by appropriate construction of $\gamma_{\theta}$. If $N \leq M(\mu)$ then we have a desired local bound on the number of $z_{j}$ 's. If $N>M(\mu)$ then, by the Weyl inequality,

$$
\begin{gathered}
\left(r_{0}-\epsilon\right)^{M(\mu)}\left(r_{0}+\mu\right)^{N-M(\mu)} \leq\left(r_{0}+\mu / 2\right)^{N} \\
\Longrightarrow \\
N \leq\left(\log \left(\frac{r_{0}+\mu}{r_{0}+\mu / 2}\right)\right)^{-1} \log \left(\frac{r_{0}+\mu}{r_{0}-\epsilon}\right) M(\mu) \leq \widetilde{C}_{\mu, \theta}\left(h^{-n}\right) .
\end{gathered}
$$

From the local bound we obtain the global bound by a covering argument, and we have proven Theorem 2. q.e.d.

Proof of Theorem 4. The proof is basically a simpler version of the proof given in Section 8 of [32], with the large part coming again from Sjöstrand's work, [23]. The only significant difference compared to [32] is the same as in the proof of Theorem 2. Therefore we restrict ourselves to an outline.

The manifold $X_{\theta}$ is a scattering manifold which differs from $X$ only on a small neighbourhood of the boundary. Hence the same construction 
of the escape function $G_{0}$ works near the boundary and the same escape function $G_{1}$ can be used near $K \subset T^{*} X \cap T^{*} X_{\theta}$ - see Section 2. Hence we obtain a global escape function, $\widetilde{G}_{\theta}$, satisfying (2.9). Let $\psi_{3} \in \mathcal{C}_{\mathrm{c}}^{\infty}\left(X_{\theta}\right)$ have the following properties

$\operatorname{supp} \psi_{3} \subset\{\psi=1\}, \quad \operatorname{Im} \sigma_{2}\left(\Delta_{\theta}\right) \geq C_{\theta}^{-1} \operatorname{Re} \sigma_{2}\left(\Delta_{\theta}\right)$ on $\operatorname{supp}\left(1-\psi_{3}\right)$, where $\psi$ in (4.8) is chosen as in the proof of Theorem 2. We then put $G_{\theta}=\psi_{3} \widetilde{G}_{\theta}$, and use it in the definition of \|\|$\|_{t G_{\theta}}$ given by (4.6) and (4.8). The point is that, where the cutoff function produces the failure of $H_{p} G_{\theta}>0$, we already have the ellipticity of the imaginary part of $\sigma_{2}\left(\Delta_{\theta}\right)$.

As in the proof of Theorem 2 we only need to consider $\psi_{1} u$ (by choosing $\psi_{1}$ and $\psi$ ), as the estimate involving $\psi_{2} u$ is the same as the proof of Theorem 2.

The proof of the following lemma follows from the proof of Lemma 8.4 of [32]. It depends on the fact that $\operatorname{Im} \sigma_{2}\left(\Delta_{\theta}\right) \uparrow_{\Lambda_{t G_{\theta}}} \geq 0$, so that (5.4) holds with $C\left(\mu+\epsilon_{0}\right)$ replaced by $C \mu$ (see Lemma 5.3 below and the properties of the escape function, $G$, in (2.9)).

Lemma 5.2. If $u \in \mathcal{C}_{\mathrm{c}}^{\infty}\left(X_{\theta}\right)$ and $\psi_{1}$ is as in the proof of Theorem 2 , then with a suitable choice of $\widetilde{\psi}$ and $\psi_{q}$ 's in Proposition 4.1, and for $t$ small enough and negative, $\mu<\mu_{0}(\theta), h<h_{0}(\theta)$,

$$
\begin{aligned}
&\left\|\mid\left(h^{2} \Delta_{\theta}-\omega_{0}\right)\left(\psi_{1} u\right)\right\|_{H\left(X_{\theta} ; \Lambda_{t G_{\theta}}, 1\right)}^{2} \\
& \geq\left(\left(r_{0}+\mu\right)^{2}-\mathcal{O}(h)\right)\left\|\mid \psi_{1} u\right\|_{H\left(X_{\theta} ; \Lambda_{t G_{\theta}}, 1\right)}^{2} \\
&-\mathcal{O}(\mu) \sum_{q=1}^{Q}\left\|f_{q, t}^{\mu} T_{q} u\right\|_{\Phi_{q, t}}^{2},
\end{aligned}
$$

where $\Phi_{q, t}$ is as in Proposition 4.1 and $f_{q, t}^{\mu}$ is the characteristic function of

$$
\pi \circ \kappa_{q}\left(\left\{\alpha \in \Lambda_{t G_{\theta}} \cap \widetilde{T^{*} Y}:\left|\sigma_{2}\left(\Delta_{\theta}\right)\right|_{\Lambda_{t G_{\theta}}}(\alpha)-\left.\omega_{0}\right|^{2} \leq\left(r_{0}+\mu\right)^{2}\right\}\right),
$$

where $\widetilde{T^{*} Y}$ is a complex neighbourhood of $T^{*} Y$ in $\widetilde{T^{*} X}, \sigma_{2}\left(\Delta_{\theta}\right)$ is the holomorphic continuation of the principal symbol of $\Delta_{\theta}$ and where

$$
\begin{array}{r}
\kappa_{q}: \widetilde{T^{*} Y} \ni\left(z,-\partial_{z} \phi_{1}(w, z)\right) \longmapsto \\
\left(w, \partial_{w} \phi_{q}(w, z)\right) \in T^{*} \mathbb{C}^{n}, \\
\phi_{q}(w, z)=\frac{i}{2}\left(\chi_{q}(z)-w\right)^{2},
\end{array}
$$

and $\pi: T^{*} \mathbb{C}^{n} \rightarrow \mathbb{C}^{n}$ is the natural projection. 
As in Section 3 of [23], for any $\epsilon>0$ there exist finite rank operators $\Xi_{q, t}^{\mu, \epsilon}$ such that for $v \in H_{\Phi_{q, t}}^{2}\left(\Omega_{q}^{\sharp}\right)$

$$
\begin{gathered}
\left\|f_{q, t}^{\mu}\left(v-\Xi_{q, t}^{\mu, \epsilon} v\right)\right\|_{\Phi_{q, t}} \leq C \epsilon\|v\|_{\Phi_{q, t}} \\
\operatorname{rank} \Xi_{q, t}^{\mu, \epsilon} \leq \min \left\{M: \operatorname{supp} f_{q, t}^{\mu} \subset \bigcup_{j=1}^{M} B\left(w_{j}, \epsilon h^{\frac{1}{2}}\right), w_{j} \in \Omega_{q}^{\sharp}\right\} .
\end{gathered}
$$

We now recall another lemma crucial in [8], [23], and [32]:

Lemma 5.3. Let $p$ denote the holomorphic extension of the principal symbol of $\Delta$ to $T^{*} \widetilde{X}$ and $p_{\theta}$ its restriction to $T^{*} X_{\theta}$. Then

$$
\begin{array}{r}
p\left(\exp t H_{G_{\theta}}^{\operatorname{Im} \sigma}(m)\right)=p_{\theta}(m)-i t H_{g_{\theta}} G_{\theta}(m)+\mathcal{O}\left(t^{2}\right)+\mathcal{O}(t \theta), \\
m \in T^{*} X_{\theta},
\end{array}
$$

where $g_{\theta}=\operatorname{Re} p_{\theta}$ and the last error term can be omitted in $T^{*} Y$ $\subset T^{*} X_{\theta} \cap T^{*} X$. Moreover, in a neighbourhood $W$ of $K$ in $S_{\epsilon}^{*} X$ we have

$$
p\left(\exp t H_{G_{\theta}}^{\operatorname{Im} \sigma}(m)\right)=p(m)-i(1+\mathcal{O}(t)) t H_{g_{\theta}} G_{\theta}(m), \quad m \in W .
$$

For the case at hand, of manifolds and irregular escape functions, the proof is given in Section 8 of [32]. Note that we took $t$ to be negative so that we guarantee a non-negative imaginary part of the symbol. q.e.d.

Using the lemma and the definitions of $f_{q, t}$ 's, $\Xi_{q, t}$ 's and of the Minkowski dimension, $m$, we conclude that for any number $\widetilde{m}>m$ we have

$$
\operatorname{rank} \Xi_{q, t}^{\mu, \epsilon} \leq C \mu^{(2 n-\widetilde{m}+1) / 2}\left(\epsilon h^{\frac{1}{2}}\right)^{-2 n} .
$$

When $K$ is of pure dimension then we can take $\widetilde{m}=m$.

Consequently Lemma 5.2 and the argument based on Lemma 5.1 give

$$
\begin{aligned}
\left\|\left(h^{2} \Delta_{\theta}-\omega_{0}\right) u\right\|_{t G_{\theta}}^{2} \geq & \left(\left(r_{0}+\mu\right)^{2}-\mathcal{O}(h)-\mathcal{O}(\epsilon \mu)\right)\|u\|_{t G_{\theta}}^{2} \\
& -\left\|Q_{\theta}^{\mu, \epsilon} u\right\|_{t G_{\theta}}^{2} \\
& \operatorname{rank} Q_{\theta}^{\mu, \epsilon} \leq C_{\epsilon} \mu^{(2 n-\widetilde{m}+1) / 2} h^{-n} .
\end{aligned}
$$

For $C h<\mu<\mu_{0} \ll_{t} 1$ we can now apply the Weyl estimates for eigenvalues in terms of characteristic values (see Section 7 of [28]) to 
obtain the bound $\mathcal{O}\left(\mu^{(2 n-\widetilde{m}+1) / 2} h^{-n}\right)$ on the number of resonances of $h^{2} \Delta$ in $|\operatorname{Re} z-1|<\mu^{\frac{1}{2}} / C,-\operatorname{Im} z>\mu$. When $\mu=C h^{1-\alpha}$ then a covering argument gives the bound $\mathcal{O}\left(h^{-\frac{1}{2}(1-\alpha)} h^{(1-\alpha)(2 n+\widetilde{m}-1) / 2} h^{-n}\right)=$ $\mathcal{O}\left(h^{-n+(1-\alpha)(2-\widetilde{m} / 2)}\right)$ on the number of resonances in

$$
|\operatorname{Re} z-1| \leq \frac{1}{2}, \quad-\operatorname{Im} z>C h^{1-\alpha}, 0 \leq \alpha \leq 1 .
$$

Another covering argument (see Section 7 of [28]) completes the proof of Theorem 4 . $\quad$ q.e.d.

\section{Appendix A}

The algebra of semiclassical scattering pseudodifferential operators is defined on any manifold $X$ with boundary. The non-semiclassical version of this calculus has been known for a long time. On $\mathbb{R}^{n}$ (identified, for our purposes, with the interior of its radial compactification) it was studied by Shubin [22], Parenti [17], and Cordes [1]; it is also the Weyl calculus for the metric

$$
\frac{|d z|^{2}}{1+|z|^{2}}+\frac{|d \xi|^{2}}{1+|\xi|^{2}}
$$

(see [10]). On manifolds it has been discussed by Schrohe [20], [21], Melrose [14], and Melrose-Zworski [16]. The approach to the calculus described below is a hybrid of those of Schrohe [20] and Melrose [14]. A different perspective on the semiclassical scattering calculus on $\mathbb{R}^{n}$ can also be found in [8].

The classical versions of all the following concepts can be recovered by setting $h=1$ throughout (and will be written without the subscript $h$ or the index indicating powers of $h$ ). We will indicate when the classical case departs from the semiclassical; otherwise, all results hold "with $h$ omitted."

We begin by defining the semiclassical scattering algebra on the ball, considered as the radial compactification of $\mathbb{R}^{n}$. We define this compactification by identifying $B^{n}$ with $S_{+}^{n}$, the upper hemisphere of the unit sphere in $\mathbb{R}^{n}$, and mapping

$$
\mathrm{RC}: z \mapsto\left(\frac{z}{\langle z\rangle}, \frac{1}{\langle z\rangle}\right) \in S_{+}^{n}
$$

(see Figure 4.) We can use $x=\left(\mathrm{RC}^{-1}\right)^{*}|z|^{-1}$ as a boundary defining function for $S_{+}^{n}$. 


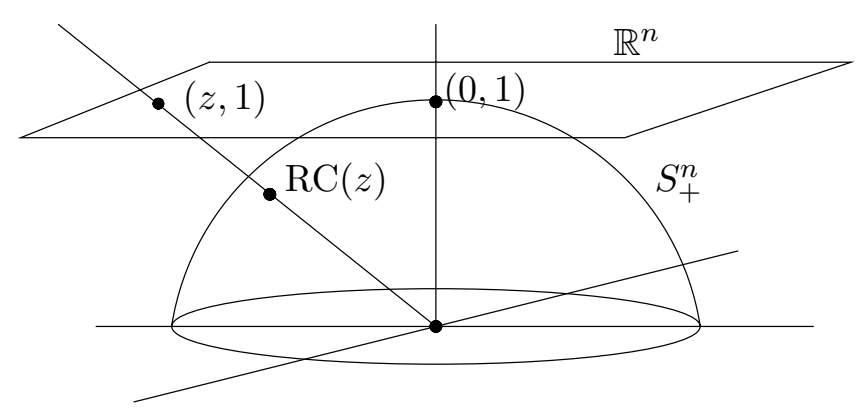

Figure 4: The radial compactification of $\mathbb{R}^{n}$

Definition A.1. A function $a \in \mathcal{C}^{\infty}\left((0,1) \times T^{*} \mathbb{R}^{n}\right)$ is a semiclassical scattering symbol of multiorder $(m, l, k)$ (we write $a \in S^{m, l, k}\left(\mathbb{R}^{n}\right)$ ) if for all multiindices $\alpha, \beta \in \mathbb{N}^{n}$, we have

$$
\left|\partial_{z}^{\alpha} \partial_{\xi}^{\beta} a\right| \leq C_{\alpha, \beta} h^{-k}\langle z\rangle^{-l-\alpha}\langle\xi\rangle^{m-\beta} .
$$

In the interests of brevity, we will restrict ourselves in this paper to the smaller class of polyhomogeneous symbols, denote $S_{\mathrm{phg}}^{m, l, k}$; these are symbols $a \in \mathcal{C}^{\infty}\left([0,1) \times T^{*} \mathbb{R}^{n}\right)$ such that

$$
a(h, z, \xi) \sim h^{-k} \sum_{i_{1}, i_{2}, i_{3}=0}^{\infty} h^{i_{1}} a_{l+i_{3}, m-i_{2}}
$$

where $a_{p, q}$ is independent of $h$ and homogeneous in $z$ and $\xi$ of order $-p$ and $q$ respectively, and the expansion means

$$
a(h, z, \xi)-h^{k} \sum_{i_{1}, i_{2}, i_{3}=1}^{N_{1}, N_{2}, N_{3}} h^{i_{1}} a_{l+i_{3}, m-i_{2}} \in S^{N_{1}-1, N_{2}+1, N_{3}+1}\left(\mathbb{R}^{n}\right) .
$$

This definition is clearer if we radially compactify $T^{*} \mathbb{R}^{n}$ in both base and fibers to obtain $S_{+}^{n} \times S_{+}^{n}$; we can use $x=1 /|z|, \sigma=1 /|\xi|$ as boundary defining functions in the respective factors. Then a (polyhomogeneous) symbol $a$ is a function of the form

$$
h^{-k} \sigma^{-m} x^{l} a_{0} \text {, where } a_{0} \in \mathcal{C}^{\infty}\left([0,1) \times S_{+}^{n} \times S_{+}^{n}\right)
$$

Definition A.2. An operator $A: \mathcal{S}\left(\mathbb{R}^{n}\right) \rightarrow \mathcal{S}^{\prime}\left(\mathbb{R}^{n}\right)$ is an element of the space $\Psi_{\mathrm{sc}, h}^{m, l, k}\left(\mathbb{R}^{n}\right)$ of (polyhomogeneous) semiclassical scattering 
pseudodifferential operators if it is given by

$$
A u(z)=\left(\frac{1}{2 \pi h}\right)^{n} \int e^{i(z-w) \cdot \xi / h} a(h, z, \xi) u(w) d w d \xi
$$

where $a \in S_{\text {phg }}^{m, l, k}\left(\mathbb{R}^{n}\right)$.

Before transferring our definition to arbitrary manifolds with boundary, we define a residual space of operators:

Definition A.3. Let $X$ be a manifold with boundary. The residual space $\Psi_{\mathrm{sc}, h}^{-\infty, \infty,-\infty}(X)$ consists of operators

$$
A: \mathcal{C}^{-\infty}(X) \rightarrow h^{\infty} \dot{\mathcal{C}}^{\infty}(X)
$$

i.e., operators with Schwartz kernels in $h^{\infty} \dot{\mathcal{C}}^{\infty}(X \times X)$. (Here the space $\dot{\mathcal{C}}^{\infty}(X)$ consists of smooth functions on $X$ vanishing to infinite order at $\partial X$, and $\mathcal{C}^{-\infty}(X)$ is the dual space of distributions; to make the pairing possible, we assume a scattering metric is fixed on $X$.)

We can consider the operators on $\mathbb{R}^{n}$ in Definition A.2 as operators on $\dot{\mathcal{C}}^{\infty}\left(S_{+}^{n}\right)$ via radial compactification. More generally, we can, by localization, extend the definition to any manifold with boundary, by the following proposition

Proposition A.4. Let $A \in \Psi_{\mathrm{sc}, h}^{m, l, k}\left(\mathbb{R}^{n}\right)$ and let $\psi: \mathbb{R}^{n} \rightarrow \mathbb{R}^{n}$ be a diffeomorphism such that $\mathrm{RC} \circ \psi \circ \mathrm{RC}^{-1}$ extends from $\left(S_{+}^{n}\right)^{\circ}$ to a diffeomorphism $S_{+}^{n} \rightarrow S_{+}^{n}$. Then

$$
\psi^{*} \circ A \circ\left(\psi^{-1}\right)^{*} \in \Psi_{\mathrm{sc}, h}^{m, l, k}\left(\mathbb{R}^{n}\right) .
$$

Proof. The following argument is mostly from [20], where the interested reader can find further detail.

Applying the Kuranishi trick of change of fiber variable, we now find that under $\psi,($ A.10) pulls back to the same expression with $a$ replaced by

$$
a\left(h, \psi(z),\left(F^{t}\right)^{-1}(z, w) \xi\right) \frac{\left|\operatorname{det} \partial \psi_{i} / \partial w_{j}\right|}{\left|\operatorname{det} F_{i j}\right|}
$$

where $F_{i j}(z, w)=\int_{0}^{1} \partial \psi_{i} / \partial z_{j}(z t+w(1-t)) d t$. $F$ and $\operatorname{det} \partial \psi / \partial w$ are clearly smooth on $S_{+}^{n} \times S_{+}^{n}$ by our assumptions on $\psi$. Furthermore, we have a polyhomogeneous expansion

$$
\psi \sim \psi_{1}+\psi_{0}+\psi_{-1}+\ldots
$$


with $\psi_{j}$ homogeneous of degree $j$; hence $\psi^{\prime \prime}(z)=O\left(|z|^{-1}\right)$, and we estimate

$$
\begin{aligned}
\left\|F_{i j}(z, w)-F_{i j}(w)\right\| & \leq C|z-w| \sup _{t \in[0,1]}\left\|\psi^{\prime \prime}(z t+w(1-t))\right\| \\
& \leq C \max \left\{|z|^{-1},|w|^{-1}\right\}|z-w|
\end{aligned}
$$

Hence if $\chi(z, w)=\phi(|z-w| /\langle z\rangle)$ with $\phi$ a smooth cutoff supported sufficiently near zero, then $F_{i j}$ is invertible on supp $\chi$. Furthermore, one can show that

$$
\begin{aligned}
\left(\frac{1}{2 \pi h}\right)^{n} \int & e^{i(z-w) \cdot \xi / h}(1-\chi(z, w)) a(h, \psi(z), \\
& \left.\left(F^{t}\right)^{-1}(z, w) \xi\right) \frac{\left|\operatorname{det} \partial \psi_{i} / \partial w_{j}\right|}{\left|\operatorname{det} F_{i j}\right|} d \xi \in \Psi_{\mathrm{sc}, h}^{-\infty, \infty,-\infty}(X)
\end{aligned}
$$

so that modulo residual operators, $\psi^{*} \circ A \circ\left(\psi^{-1}\right)^{*}$ has Schwartz kernel

$$
\left(\frac{1}{2 \pi h}\right)^{n} \int e^{i(z-w) \cdot \xi / h} \chi(z, w) a\left(h, \psi(z),\left(F^{t}\right)^{-1}(z, w) \xi\right) \frac{\left|\operatorname{det} \partial \psi_{i} / \partial w_{j}\right|}{\left|\operatorname{det} F_{i j}\right|} d \xi
$$

Modulo a further residual error, we can rewrite this in the form

$$
\left(\frac{1}{2 \pi h}\right)^{n} \int e^{i(z-w) \cdot \xi / h} \widetilde{a}(h, z, \xi) d \xi
$$

in the usual manner, setting

$$
b=\chi(z, w) a\left(h, \psi(z),\left(F^{t}\right)^{-1}(z, w) \xi\right) \frac{\left|\operatorname{det} \partial \psi_{i} / \partial w_{j}\right|}{\left|\operatorname{det} F_{i j}\right|}
$$

and

$$
\left.\widetilde{a}(h, z, \xi) \sim \sum_{\alpha} \frac{h^{|\alpha|} i^{-|\alpha|}}{\alpha !} \partial_{\xi}^{\alpha} \partial_{w}^{\alpha} b(h, z, w, \xi)\right|_{z=w}
$$

the appearance of $\chi$ in the definition of $b$ is irrelevant to the definition of $\widetilde{a}$ owing to the restriction to the diagonal; the same is true of the Jacobian factors. The $\psi_{i}$ 's and therefore $F_{i j}$ are polyhomogeneous; hence the symbol $\tilde{a}$ is also polyhomogeneous. $\quad$ q.e.d. 
Definition A.5. Let $X$ be a manifold with boundary. Then an operator $A: \dot{\mathcal{C}}^{\infty}(X) \rightarrow \mathcal{C}^{-\infty}(X)$ is in $\Psi_{\mathrm{sc}, h}^{m, l, k}(X)$ if for every $\phi_{1}, \phi_{2}$ with $\operatorname{supp} \phi_{1} \cap \operatorname{supp} \phi_{2}=\emptyset$, we have

$$
\phi_{1} A \phi_{2} \in \Psi_{\mathrm{sc}, h}^{-\infty, \infty,-\infty}(X),
$$

and on the other hand, if $\phi_{1}$ and $\phi_{2}$ have support in an open set $U \subset X$ such that there is a diffeomorphism $\psi: U \rightarrow V \subset S_{+}^{n}$,

$$
(\mathrm{RC})^{*}\left(\psi^{-1}\right)^{*} \phi_{1} A \phi_{2}(\psi)^{*}\left(\mathrm{RC}^{-1}\right)^{*} \in \Psi_{\mathrm{sc}, h}^{m, l, k}\left(\mathbb{R}^{n}\right) .
$$

These operators form a calculus:

Proposition A.6. Let $A \in \Psi_{\mathrm{sc}, h}^{m, l, k}(X)$ and $B \in \Psi_{\mathrm{sc}, h}^{m^{\prime}, l^{\prime}, k^{\prime}}(X)$ then $A B \in \Psi_{\mathrm{sc}, h}^{m+m^{\prime}, l+l^{\prime}, k+k^{\prime}}(X)$ and $A^{*} \in \Psi_{\mathrm{sc}, h}^{m, l, k}(X)$.

(The proof proceeds just as with the ordinary Kohn-Nirenberg calculus; for the explicit symbol computations on $\mathbb{R}^{n}$, see Section 7 of [3].)

There is a principal symbol map for the semiclassical scattering calculus that contains more information than just the ordinary principal symbol of the pseudodifferential operator over $X^{\circ}$; the symbol measures how the operator behaves under conjugation by oscillatory distributions near $\partial X$ (the "boundary at infinity") as well as by distributions supported in $X^{\circ}$ with oscillatory Fourier transform (this is the traditional notion of principal symbol). We begin by describing where the symbol lives:

Definition A.7. Let $\mathcal{V}_{b}(X)$ denote the Lie algebra of vector fields on $X$, tangent to $\partial X$. Let ${ }^{\text {sc }} T X$ be the vector bundle on $X$ whose sections lie in $x \mathcal{V}_{b}$. Let ${ }^{\mathrm{sc}} T^{*} X$ be its dual bundle. Let ${ }^{\mathrm{sc}} \bar{T}^{*} X$ denote the fiber radial compactification of ${ }^{s c} T^{*} X$ (it is a manifold with corners). Let ${ }^{\mathrm{sc}} S^{*} X$ be the unit cosphere bundle of ${ }^{\mathrm{sc}} T^{*} X$, considered as the fiber boundary of ${ }^{\mathrm{sc}} \bar{T}^{*} X$.

Definition A.8. Let $A \in \Psi_{\mathrm{sc}, h}^{m, l, k}\left(S_{+}^{n}\right)$ be given by

$$
A u(z)=\left(\frac{1}{2 \pi h}\right)^{n} \int e^{i(z-w) \cdot \xi / h} a(h, z, \xi) u(w) d w d \xi
$$

(in Euclidean coordinates) with $a \in S_{\mathrm{phg}}^{m, l, k}\left(\mathbb{R}^{n}\right)$. Let $\left(\mathrm{RC}^{-1} \times \mathrm{RC}^{-1}\right)^{*} a$ denote the pullback of $a$ to $h^{-k} \sigma^{-m} x^{l}$ times a smooth function on 
$[0,1) \times S_{+}^{n} \times S_{+}^{n}$ Then the symbol of $A$ is defined as

$$
\begin{aligned}
\sigma_{\mathrm{sc}, h}^{m, l, k}(A) & =\left(\mathrm{RC}^{-1} \times \mathrm{RC}^{-1}\right)^{*} a \\
& \in \frac{h^{-k} \sigma^{-m} x^{l} \mathcal{C}^{\infty}\left([0,1) \times S_{+}^{n} \times S_{+}^{n}\right)}{h^{-k+1} \sigma^{-m+1} x^{l+1} \mathcal{C} \infty\left([0,1) \times S_{+}^{n} \times S_{+}^{n}\right) .}
\end{aligned}
$$

Loosely speaking (i.e., forgetting about overall powers of defining functions), we can think of the symbol as the restriction of $\left(\mathrm{RC}^{-1} \times \mathrm{RC}^{-1}\right)^{*} a$ to the boundary of $[0,1) \times S_{+}^{n} \times S_{+}^{n}$. The part of the symbol defined in $[0,1) \times\left(S_{+}^{n}\right)^{\circ} \times \partial S_{+}^{n}$ is just the ordinary principal symbol of $A$ as a pseudodifferential operator on $\mathbb{R}^{n}$. Note that $[0,1) \times S_{+}^{n} \times S_{+}^{n}$ is a manifold with corners with three codimension-one boundary faces (unless $n=1$ ).

The manifold $S_{+}^{n} \times S_{+}^{n}$ should really be thought of as ${ }^{\mathrm{sc}} \bar{T}^{*} S_{+}^{n}$, as is indicated by

Proposition A.9. Definition A.8 makes invariant sense, i.e., $\sigma_{\mathrm{sc}, h}^{m, l, k}(A)$ is an invariantly defined element of

$$
h^{-k} \sigma^{-m} x^{l} \mathcal{C}^{\infty}\left([0,1) \times{ }^{\mathrm{sc}} \bar{T}^{*} S_{+}^{n}\right) / h^{-k+1} \sigma^{-m+1} x^{l+1} \mathcal{C}^{\infty}\left([0,1) \times{ }^{\mathrm{sc}} \bar{T}^{*} S_{+}^{n}\right) .
$$

Proof. Evaluate (A.11) at $z=w$ and insert it into the $\alpha=0$ term of (A.12); the other terms vanish at each of the three boundary faces $h=0, x=0, \sigma=0$. q.e.d.

As a result of Propositions A.4 and A.9, the definition of $\sigma_{\mathrm{sc}, h}$ can be extended to a map

$$
\begin{aligned}
& \sigma_{\mathrm{sc}, h}^{m, l, k}: \Psi_{\mathrm{sc}, h}^{m, l, k}(X) \\
& \rightarrow h^{-k} \sigma^{-m} x^{l} \mathcal{C}^{\infty}\left([0,1) \times{ }^{\mathrm{sc}} \bar{T}^{*} X\right) / h^{-k+1} \sigma^{-m+1} x^{l+1} \mathcal{C}^{\infty}\left([0,1) \times{ }^{\mathrm{sc}} \bar{T}^{*} X\right),
\end{aligned}
$$

where $X$ is a manifold with boundary.

Example . Let $\Delta$ be the Laplacian with respect to a scattering metric $g$ on $S_{+}^{n}$. Let $\Delta$ be the corresponding Laplace-Beltrami operator. Then $\Delta \in \Psi_{\mathrm{sc}}^{2,0}(X)$ and $h^{2} \Delta \in \Psi_{\mathrm{sc}, h}^{2,0,0}(X)$. We have

$$
\sigma_{\mathrm{sc}, h}^{2,0}\left(h^{2} \Delta\right)=g(z, \xi)=\sigma_{\mathrm{sc}}^{2,0,0}(\Delta),
$$

where we abuse notation, confusing $g(z, \xi)$ with its restriction to the various boundary faces in question. See [14]. 
The semiclassical scattering symbol has all the desirable properties of a commutative symbol:

Proposition A.10. The symbol map is multiplicative: if $A \in \Psi_{\mathrm{sc}, h}^{m, l, k}(X)$ and $B \in \Psi_{\mathrm{sc}, h}^{m^{\prime}, l^{\prime}, k^{\prime}}(X)$, then

$$
\sigma_{\mathrm{sc}, h}^{m+m^{\prime}, l+l^{\prime}, k+k^{\prime}}(A B)=\sigma_{\mathrm{sc}, h}^{m, l, k}(A) \sigma_{\mathrm{sc}, h}^{m^{\prime}, l^{\prime}, k^{\prime}}(B),
$$

and $\sigma_{\mathrm{sc}, h}^{m, l, k}\left(A^{*}\right)=\overline{\sigma_{\mathrm{sc}, h}^{m, l, k}(A)}$.

There is a short exact sequence

$$
\begin{aligned}
0 & \rightarrow \Psi_{\mathrm{sc}, h}^{m-1, l+1, k-1}(X) \rightarrow \Psi_{\mathrm{sc}, h}^{m, l, k}(X) \\
& \stackrel{\sigma_{\mathrm{sc}, h}^{m, l}}{\longrightarrow} \frac{h^{-k} \sigma^{-m} x^{l} \mathcal{C}^{\infty}\left([0,1) \times{ }^{\mathrm{sc}} \bar{T}^{*} X\right)}{h^{-k+1} \sigma^{-m+1} x^{l+1} \mathcal{C}^{\infty}\left([0,1) \times{ }^{\mathrm{sc}} \bar{T}^{*} X\right)} \rightarrow 0 .
\end{aligned}
$$

That $\sigma_{\mathrm{sc}, h}$ is the "correct" notion of symbol is demonstrated by

Proposition A.11. If $A \in \Psi_{\mathrm{sc}, h}^{m, l, k}(X)$ is elliptic in the sense that $\sigma_{\mathrm{sc}, h}^{m, l, k}(A)$ does not vanish on $\partial\left([0,1) \times{ }^{\mathrm{sc}} \bar{T}^{*} X\right)$ then there exists a parametrix $B \in \Psi_{\mathrm{sc}, h}^{-m,-l,-k}(X)$ such that

$$
A B-I d, B A-I d \in \Psi_{\mathrm{sc}, h}^{-\infty, \infty,-\infty}(X) .
$$

If $\sigma_{\mathrm{sc}, h}^{m, l, k}(A)$ does not vanish on $0 \times{ }^{\mathrm{sc}} \bar{T}^{*} X$ then the same is true for $h$ sufficiently small.

If $\sigma_{\mathrm{sc}, h}^{m, l, k}(A)$ does not vanish on $[0,1) \times \partial^{\mathrm{sc}} \bar{T}^{*} X$ then there exists $B \in \Psi_{\mathrm{sc}, h}^{-m,-l,-k}(X)$ such that

$$
A B-I d, B A-I d \in \Psi_{\mathrm{sc}, h}^{-\infty, \infty}(X) .
$$

The proof is the usual iterative argument.

Remark. In the classical case, the symbol is a function only on $\partial\left({ }^{\mathrm{sc}} \bar{T}^{*} X\right)$. Nonvanishing of $\sigma_{\mathrm{sc}}(A)$ on $\partial\left({ }^{\mathrm{sc}} \bar{T}^{*} X\right)$ ensures invertibility of $A$ modulo $\Psi_{\mathrm{sc}}^{-\infty, \infty}(X)$.

We now introduce a scale of Sobolev spaces adapted to the semiclassical scattering calculus.

Definition A.12. Let $L_{\mathrm{sc}}^{2}(X)$ denote the space of distributions on $X$ that are square-integrable with respect to a scattering metric, and 
let $L_{\mathrm{sc}, h}^{2}(X)=\mathcal{C}\left([0,1) ; L_{\mathrm{sc}}^{2}(X)\right)$. Set

$$
\begin{aligned}
& H_{\mathrm{sc}, h}^{m, l}(X) \\
& =\left\{u \in \mathcal{C}\left([0,1) ; \mathcal{C}^{-\infty}(X)\right): x^{-l}\left(\mathrm{Id}+h^{2} \Delta\right)^{m / 2} u \in \mathcal{C}\left([0,1) ; L_{\mathrm{sc}}^{2}(X)\right)\right\}
\end{aligned}
$$

and

$$
H_{\mathrm{sc}}^{m, l}(X)=\left\{u \in \mathcal{C}^{-\infty}(X): x^{-l}(\mathrm{Id}+\Delta)^{m / 2} u \in L_{\mathrm{sc}}^{2}(X)\right\}
$$

with $x$ a boundary defining function and $\Delta$ the Laplacian with respect to a scattering metric.

Proposition A.13. Let $A \in \Psi_{\mathrm{sc}, h}^{m, l, k}(X)$ and $p, q \in \mathbb{R}$. Then

$$
A=\mathcal{O}\left(h^{-k}\right): H_{\mathrm{sc}, h}^{p, q}(X) \rightarrow H_{\mathrm{sc}, h}^{p-m, q+l}(X) .
$$

Let $B \in \Psi_{\mathrm{sc}}^{m, l}(X)$. Then

$$
B: H_{\mathrm{sc}}^{p, q}(X) \rightarrow H_{\mathrm{sc}}^{p-m, q+l}(X)
$$

is bounded.

The inclusions $H_{\mathrm{sc}, h}^{p, q}(X) \subset H_{\mathrm{sc}, h}^{p^{\prime}, q^{\prime}}(X)$ and $H_{\mathrm{sc}}^{p, q}(X) \subset H_{\mathrm{sc}}^{p^{\prime}, q^{\prime}}(X)$ are compact if $p<p^{\prime}, q, q^{\prime}$.

The proof is by the usual Hörmander square root method [10] to show boundedness on $L^{2}$, together with the basic properties of the calculus. Compact embedding of weighted Sobolev spaces is also standard.

\section{Appendix B (with Edith Mooers)}

The complex scaling method can be applied efficiently in many concrete situations - see references in [19]. In the simplest case, when there is a single hyperbolic orbit, numerical computations show the accuracy of the semiclassical results recalled in the Example in Section 2. We now briefly describe the numerical method used. We stress that we cannot compute the resonances explicitly by the special function methods which are available for the case of the hyperbolic cylinder (see [7] and references therein)

The Laplacian (2.10) decomposes into a family of one-dimensional operators depending on the spectral parameter $n$ of the circle. The resonances for each of these operators are computed by first applying 


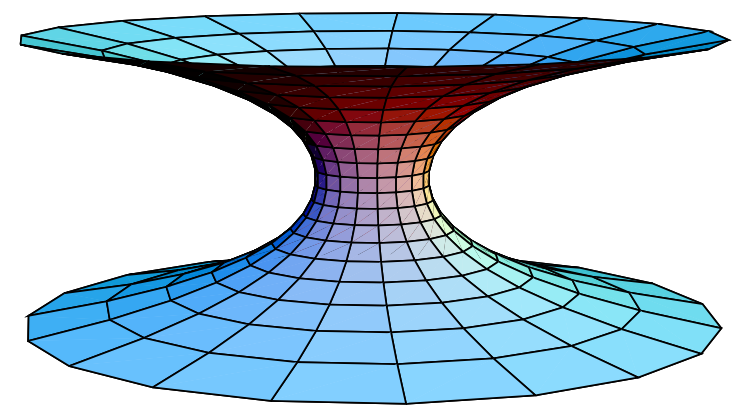

Figure 5: The catenoid.

complex scaling (a substitution $r=r^{\prime} e^{i \theta}$ ) in one dimension, and then reducing the problem to finite dimension by truncating a basis of $L^{2}(\mathbb{R})$. The simplest basis to choose, and one particularly well-adapted to this problem (because of the presence of a non-degenerate potential maximum), is that of eigenfunctions of the quantum harmonic oscillator.

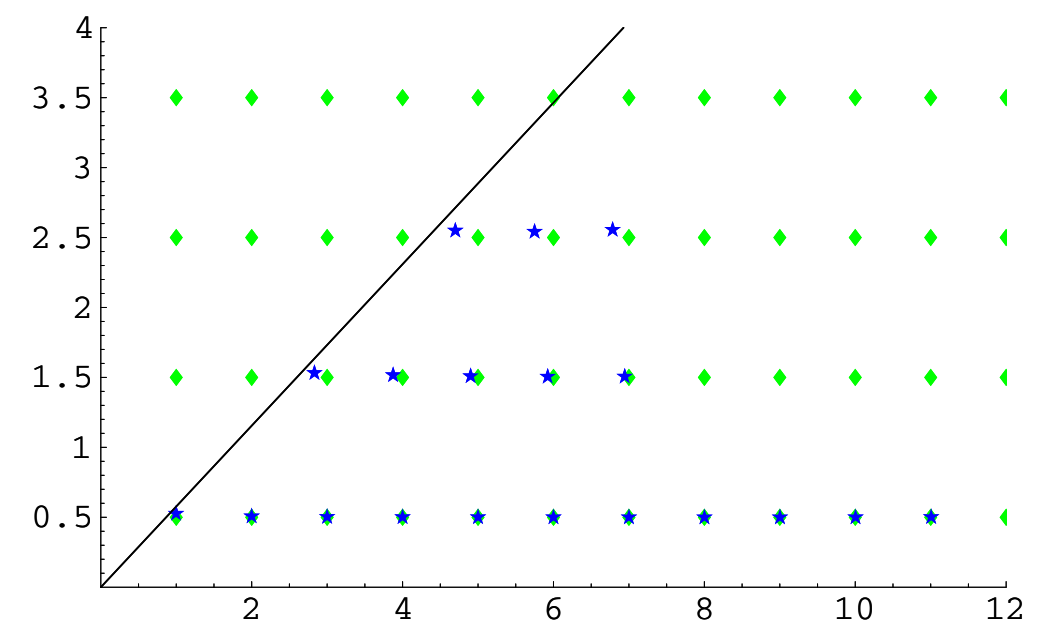

Figure 6: Resonances for the catenoid with $a=1$, and the lattice given by the first terms of the semiclassical approximation (2.11).

This program was implemented in Mathematica, ${ }^{\complement}$ using the first 50 
of the built-in Hermite functions. Matrix elements for the complexscaled operators were computed numerically, and the eigenvalues of the resulting nonselfadjoint matrix were then evaluated. Most of these eigenvalues correspond to the rotated continuous spectrum. Computations for several angles of complex scaling were compared in order to sift out eigenvalues which correspond closely to actual resonances.

\section{References}

[1] H. O. Cordes, , A global parametrix for pseudodifferential operators over $\mathbb{R}^{n}$ with applications, Preprint No. 90, SFB 72, Bonn, 1976.

[2] H. L. Cycoon, R. G. Froese, W. Kirsch \& B. Simon, Schrödinger operators, Springer, 1987.

[3] M. Dimassi \& J. Sjöstrand, Spectral asymptotics in the semi-classical limit, Lecture notes, to appear.

[4] J. Edward, Bounds on resonances for the Laplacian on perturbations of quotients of $\mathbf{R}^{n}$ by a finite subgroup of $G L(n)$, Preprint, February 1999.

[5] C. Gérard \& J. Sjöstrand, Semiclassical resonances generated by a closed trajectory of hyperbolic type, Comm. Math. Phys. 108 (1987) 391-421.

[6] H. Grauert, On Levi's problem and the imbedding of real-analytic manifolds, Ann. of Math. 68 (1958) 460-472.

[7] L. Guillopé \& M. Zworski, Scattering asymptotics for Riemann surfaces. Ann. of Math. 145 (1997) 597-660.

[8] B. Helffer \& J. Sjöstrand, Résonances en limite semi-classique. [Resonances in the semi-classical limit] Mémoires de la S.M.F. 114 (3) (1986).

[9] P. Hislop \& I.M. Sigal, Introduction to spectral theory with applications to Schrödinger operators, Appl. Math. Sci., Springer, Vol. 113, 1996.

[10] L. Hörmander, The analysis of linear partial operators III, Springer, Berlin, 1985.

[11] L. Hörmander, An Introduction to complex analysis in several variables, North Holland, Amsterdam-London, 1973.

[12] N. Kapouleas, Complete embedded minimal surfaces of finite total curvature, J. Differential Geom. 47 (1997) 95-169.

[13] K. Lin, Computation of resonances in semi-classical chaotic scattering, in preparation. 
[14] R. B. Melrose, Spectral and scattering theory for the Laplacian on asymptotically Euclidian spaces, Spectral and Scattering Theory, M. Ikawa, ed. Marcel Dekker, 1994.

[15] R. B. Melrose, Geometric scattering theory, Cambridge University Press, 1995.

[16] R.B. Melrose \& M. Zworski, Scattering metrics and geodesic flow at infinity, Invent. Math. 124 (1996) 389-436.

[17] C. Parenti, Operatori pseudodifferentiali in $\mathbb{R}^{n}$ e applicazioni, Ann. Math. Pura Appl. 93 (1972) 359-389.

[18] G. Popov, Quasimodes for the Laplace operator and glancing hypersurfaces, Microlocal Analysis and Non-linear Waves, Minnesota, 1989, (M. Beals, R. Melrose and J. Rauch eds.), Springer, Berlin, 1991.

[19] A. Sá Barreto \& M. Zworski, Distribution of resonances for spherical black holes, Math. Res. Lett. 4 (1997) 103-121.

[20] E. Schrohe, Spaces of weighted symbols and weighted Sobolev spaces on manifolds, Pseudodifferential operators, Proceedings, Oberwolfach 1986, Lecture Notes in Math. 1256, Springer, Berlin, 1987.

[21] Complex powers on noncompact manifolds and manifolds with singularities, Math. Ann. 281 (1988) 393-409.

[22] M. A. Shubin, Pseudodifferential operators in $\mathbb{R}^{n}$, Dokl. Akad. Nauk SSSR 196 No. 2 (1971) 316-319; Soviet Math. Dokl. 12 No.1 (1971) 147-151.

[23] J. Sjöstrand, Geometric bounds on the density of of resonances for semi-classical problems, Duke Math. J. 60 (1990) 1-57.

[24] Density of resonances for strictly convex analytic obstacles, Canad. J. Math. 48 (1996) 397-447.

[25] _ A Trace Formula and Review of Some Estimates for Resonances, Microlocal analysis and spectral theory (Lucca, 1996), 377-437, NATO Adv. Sci. Inst. Ser. C Math. Phys. Sci., 490, Kluwer Acad. Publ., Dordrecht, 1997.

[26] _ Resonances for bottles and trace formulae, Preprint, June, 1998.

[27] J. Sjöstrand \& M. Zworski, Complex scaling and the distribution of scattering poles, J. Amer. Math. Soc. 4 (1991) 729-769.

[28] - The complex scaling method of scattering by strictly convex obstacles, Ark. Math. 33 (1995) 135-172.

[29] P. Stefanov, Quasimodes and resonances: sharp lower bounds, Duke Math. J. 99 (1999) 75-92.

[30] S. H. Tang \& M. Zworski, From quasi-modes to resonances. Math. Res. Lett. 5 (1998) 261-272. 
[31] J. Wunsch \& M. Zworski, The FBI transform on compact $\mathcal{C}^{\infty}$ manifolds, Trans. Amer. Math. Soc. 353 (2001) 1151-1167.

[32] M. Zworski, Dimension of the limit set and the density of resonances for convex co-compact hyperbolic surfaces, Invent. Math. 136 (2) (1999) 353-409.

[33] $\longrightarrow$ Resonances in physics and geometry, Notices of Amer. Math. Soc. 46 (3) (1999) 319-328.

\author{
University of California, Berkeley \\ SUNY, STONY BROOK, NY
}

